\title{
McCammond's normal forms for free aperiodic semigroups revisited
}

\author{
J. Almeida, J. C. Costa and M. Zeitoun
}

\begin{abstract}
This paper revisits the solution of the word problem for $\omega$-terms interpreted over finite aperiodic semigroups, obtained by J. McCammond. The original proof of correctness of McCammond's algorithm, based on normal forms for such terms, uses McCammond's solution of the word problem for certain Burnside semigroups. In this paper, we establish a new, simpler, correctness proof of McCammond's algorithm, based on properties of certain regular languages associated with the normal forms. This method leads to new applications.
\end{abstract}

\section{Introduction}

An $\omega$-term is a formal expression obtained from letters of an alphabet $X$ using two operations: the binary, associative concatenation and the unary $\omega$-power. Any $\omega$-term $\alpha$ can be given a natural interpretation on a finite semigroup $S$ as a mapping $\alpha_{S}: S^{X} \rightarrow S$, as follows: each letter $x$ of $X$ is interpreted as the mapping sending each element of $S^{X}$ to its image on $x$, the concatenation is viewed as the semigroup multiplication, while the $\omega$-power is interpreted as the unary operation which sends each element of $S$ to its unique idempotent power. The $\omega$-word problem for a class $\mathcal{C}$ of finite semigroups consists in deciding whether two $\omega$-terms have the same interpretation over every semigroup of $\mathcal{C}$.

One motivation for considering the $\omega$-word problem is that its decidability is one of the requirements of a property of pseudovarieties (classes of finite semigroups closed under taking subsemigroups, homomorphic images, and finite direct products) called tameness, introduced by the first author and Steinberg $[\mathbf{3}, \mathbf{1 0}, \mathbf{1 1}]$ to solve the decidability problem for iterated semidirect products of pseudovarieties. In spite of its limitations for that purpose under current knowledge, tameness remains a property of interest which has also been used to solve membership problems involving other types of operators [6] (see [7] and [26, §3.7.3] for a discussion). A difficult problem occurring in computer science is related to a weak form of tameness [4]. It asks if it is possible to separate two given regular languages by a language recognized by a semigroup of a given pseudovariety. For the pseudovariety A of all aperiodic (or group-free) semigroups, recognizing exactly first-order definable languages [23, 27], it amounts to finding a first-order formula holding on (all words of) one language, and whose negation

Received 15 May 2014; revised 24 September 2014.

2010 Mathematics Subject Classification 20M05, 20M07 (primary), 20M35, 68Q70 (secondary).

This work was partly supported by the PESSOA French-Portuguese project Egide-Grices 11113YM Automata, profinite semigroups and symbolic dynamics. The work leading to this paper was also carried out within the framework of the ESF programme 'Automata: from Mathematics to Applications (AutoMathA)', whose support is gratefully acknowledged. The work of the first and second authors was supported, in part, by the European Regional Development Fund, through the programme COMPETE, and by the Portuguese Government through FCT - Fundação para a Ciência e a Tecnologia, respectively under the projects PEst-C/MAT/UI0144/2013 and PEst-OE/MAT/UI0013/2014. Both the first and second authors were also supported by FCT through the project PTDC/MAT/65481/2006, which was partly funded by the European Community Fund FEDER. The work of the third author was partially supported by ANR 2010 BLAN 020201 FREC. 
holds on the other one. It was solved algebraically by Henckell [17], and by simple combinatorial methods by Place and the third author [25].

The $\omega$-word problem has been solved for some pseudovarieties. The case of the pseudovariety of all $\mathcal{J}$-trivial semigroups, solved by the first author in $[\mathbf{1}]$, constitutes a classical example. Another remarkable example, achieved by McCammond [22], is given by the pseudovariety A. Recently, an alternative algorithm for deciding the $\omega$-word problem for $\mathrm{A}$ has been proposed in [18]. It is based on Ehrenfeucht-Fraïssé games played on representations of $\omega$-words, and this approach makes it possible to obtain an EXPTIME upper bound for this decision problem. One should note, however, that the correctness proof of this new algorithm itself relies on McCammond's algorithm.

The $\omega$-word problem has been solved for other pseudovarieties. It has been obtained by the second author $[\mathbf{1 3}]$ for the pseudovariety of local semilattices. The first and third authors [12] solved the $\omega$-word problem for the pseudovariety of $\mathcal{R}$-trivial semigroups, and their techniques have been adapted for the pseudovariety DA, which consists of all finite semigroups whose regular $\mathcal{J}$-classes are aperiodic semigroups [24]. Recently, the second author [14] has applied techniques similar to those of this paper to show decidability of the $\omega$-word problem for the pseudovariety of all finite semigroups.

Unlike the cases of local semilattices $[\mathbf{1 5}, \mathbf{1 6}]$ and $\mathcal{R}$-trivial semigroups $[\mathbf{6}, \mathbf{7}]$, there is as yet no published proof of tameness of $A$, but the above-mentioned solution of the $\omega$-word problem for A is a step forward in that direction. McCammond's solution [22] consists in the reduction of arbitrary $\omega$-terms to a certain normal form. McCammond then goes on to show that different $\omega$-terms in normal form cannot have the same interpretation over $A$, which he does by invoking his results on free Burnside semigroups [21].

In this paper we give an alternative proof of McCammond's normal form theorem for $\omega$ terms over $\mathrm{A}$, which is independent of the theory of free Burnside semigroups. Our approach consists in associating to each $\omega$-term $\alpha$ a decreasing sequence of regular languages $\left(L_{n}[\alpha]\right)_{n}$, whose key property is that, if $\alpha$ is in McCammond's normal form, then $L_{n}[\alpha]$ is ultimately star-free. Another crucial element in the proof is the fact that if $\alpha$ and $\beta$ are $\omega$-terms in normal form and $L_{n}[\alpha] \cap L_{n}[\beta] \neq \emptyset$ for all $n$, then $\alpha=\beta$.

This new approach, and particularly the fact that the languages $L_{n}[\alpha]$ are star-free, also yields new applications on the structure of the free pro-A semigroup. Some elements of this semigroup, called $\omega$-words, have a nice form: they can actually be represented by an $\omega$-term. We show that in the free pro-A semigroup, every factor of an $\omega$-word is also an $\omega$-word. In turn this result is a central piece in [9], whose main result provides a characterization of $\omega$-words in the free pro-A semigroup.

The paper is organized as follows. In $\S 2$ we review background material, including the description of McCammond's normal form. We introduce term expansions and the languages $L_{n}[\alpha]$ in $\S 3$, and we prove some of their basic properties. Section 4 is mainly devoted to the proof of a combinatorial and central lemma, about $\omega$-terms whose $\omega$-powers are not nested. In $\S 5$ we present the main properties of the languages $L_{n}[\alpha]$ and the alternative proof of uniqueness of McCammond's normal forms for $\omega$-terms over A. In $\S 6$ we establish the starfreeness of $L_{n}[\alpha]$ for $\alpha$ in normal form and $n$ large enough. Finally, we investigate in $\S 7$ other properties of the languages $L_{n}[\alpha]$, and derive some applications.

\section{Preliminaries}

In this section we briefly recall the basic definitions and results that will be used throughout the paper. The reader is referred to $[\mathbf{2}, \mathbf{2 6}]$ for general background, and to [5] for a quick introduction to the classical theories of pseudovarieties, regular languages and profinite semigroups. For further details about combinatorics on words, see [19, 20]. 


\subsection{Words}

In the following, $X$ denotes a finite nonempty alphabet. The free semigroup (respectively, the free monoid) generated by $X$ is denoted by $X^{+}$(respectively, by $X^{*}$ ). The length of a word $u \in X^{*}$ is denoted by $|u|$. Given words $u$ and $v$, we write $u \preccurlyeq v$ if $u$ is a prefix of $v$ and $u \prec v$ if $u \preccurlyeq v$ and $u \neq v$. If $v=u w$, we denote by $u^{-1} v$ the suffix $w$ of $v$. When $w=x y z=x^{\prime} y^{\prime} z^{\prime}$, we say that the factors $y$ and $y^{\prime}$ of $w$ are synchronized in $w$ if $x=x^{\prime}$ and $z=z^{\prime}$ (whence $y=y^{\prime}$ ). They overlap if $x \preccurlyeq x^{\prime} \prec x y$ or $x^{\prime} \preccurlyeq x \prec x^{\prime} y^{\prime}$. They overlap on (at least) $k>0$ positions if in addition $y=u_{1} v u_{2}$ and $y^{\prime}=u_{1}^{\prime} v^{\prime} u_{2}^{\prime}$ where $|v|=\left|v^{\prime}\right|=k$ and $v, v^{\prime}$ are synchronized in $w$.

The following result is known as Fine and Wilf's theorem (see $[\mathbf{1 9}, \mathbf{2 0}]$ ).

Proposition 2.1 (Fine and Wilf's theorem). Let $u, v \in X^{+}$. If two powers $u^{k}$ and $v^{\ell}$ of $u$ and $v$ have a common prefix of length at least $|u|+|v|-\operatorname{gcd}(|u|,|v|)$, then $u$ and $v$ are powers of the same word.

A primitive word is a word that cannot be written in the form $u^{n}$ with $n>1$. Two words $w$ and $z$ are conjugate if one can write $w=u v$ and $z=v u$, where $u, v \in X^{*}$. All conjugates of a primitive word are also primitive. Let an order be fixed for the letters of the alphabet $X$. A Lyndon word is a primitive word that is minimal, with respect to the lexicographic ordering, in its conjugacy class. We recall a property following from [19, Proposition 5.1.2].

Lemma 2.2. If $t \in X^{*}$ is both a prefix and a suffix of a Lyndon word $w$, then either $t$ is the empty word, or $t$ is the word $w$ itself.

\subsection{Pseudowords and $\omega$-words}

In this paper, we deal with the pseudovariety A of all finite aperiodic, or group-free, semigroups. These are the finite semigroups $T$ for which there exists some integer $n>0$ such that $s^{n}=s^{n+1}$ for every $s \in T$. We write $S$ for the class of all finite semigroups.

Given a pseudovariety $\mathrm{V}$, we denote by $\bar{\Omega}_{X} \vee$ the free pro- $\mathrm{V}$ semigroup over $X$ (see [5] for its construction and main properties). We briefly recall here some of its properties needed in the paper. First, $\bar{\Omega}_{X} \vee$ is a compact topological semigroup whose elements are called pseudowords over $\mathrm{V}$. For $\mathrm{V}=\mathrm{S}$ or $\mathrm{A}$, the free semigroup $X^{+}$embeds in $\bar{\Omega}_{X} \mathrm{~V}$ and is dense in $\bar{\Omega}_{X} \mathrm{~V}$. For $L \subseteq X^{+}$, we denote by $\operatorname{cl}(L)$ (respectively, $\operatorname{cl}_{\mathrm{A}}(L)$ ) its closure in $\bar{\Omega}_{X} \mathrm{~S}$ (respectively, in $\bar{\Omega}_{X} \mathrm{~A}$ ). There is a unique continuous homomorphism from $\bar{\Omega}_{X} S$ to $\bar{\Omega}_{X} \mathrm{~A}$ sending each $x \in X$ to itself, and we denote it by $p_{\mathrm{A}}$. Note that $p_{\mathrm{A}}(\operatorname{cl}(L))=\operatorname{cl}_{\mathrm{A}}(L)$.

Given $z \in \bar{\Omega}_{X} \vee$, the closed subsemigroup of $\bar{\Omega}_{X} \vee$ generated by $z$ contains a single idempotent denoted by $z^{\omega}$, which is the limit of the sequence $z^{n !}$. Note that $z z^{\omega}=z^{\omega} z$. We set $z^{\omega+1}=z z^{\omega}$. In $\bar{\Omega}_{X} \mathrm{~A}$, we have $z^{\omega+1}=z^{\omega}$. For $\alpha, \beta \in \bar{\Omega}_{X} \mathrm{~S}$, we say that A satisfies $\alpha=\beta$ if $p_{\mathrm{A}}(\alpha)=p_{\mathrm{A}}(\beta)$. For example, A satisfies $z^{\omega+1}=z^{\omega}$ for all $z \in \bar{\Omega}_{X}$ S.

A unary semigroup is an algebra $(S, \cdot, \tau)$, with - binary and associative and $\tau$ unary. A free pro- $V$ semigroup has a natural structure of unary semigroup, where $\tau$ is interpreted as the $\omega$ power. We denote by $\Omega_{X}^{\omega} \vee$ the unary subsemigroup of $\bar{\Omega}_{X} \vee$ generated by $X$, whose elements are called $\omega$-words over $\mathrm{V}$. Each $\omega$-word has a representation by a formal term over $X$ in the signature $\{\cdot, \omega\}$, called an $\omega$-term. We do not distinguish between $\omega$-terms that only differ in the order in which multiplications are to be carried out. Finally, let $\mathcal{T}_{X}$ be the unary semigroup of $\omega$-terms, which is freely generated by $X$ as a unary semigroup. Sometimes, it will be useful to consider also the empty $\omega$-term, which is identified with the empty word.

\subsection{The $\omega$-word problem for $\mathrm{A}$}

McCammond [22] represents $\omega$-terms over $X$ as nonempty well-parenthesized words over the alphabet $Y=X \uplus\{()$,$\} , which do not have () as a factor. The \omega$-term associated with such 
a word is obtained by replacing each matching pair of parentheses $(*)$ by $(*)^{\omega}$. For example, the parenthesized word $((a) b)$ represents the $\omega$-term $\left(a^{\omega} b\right)^{\omega}$. Conversely, every $\omega$-term over $X$ determines a unique well-parenthesized word over $Y$. We identify $\mathcal{T}_{X}$ with the set of these wellparenthesized words over $Y$. Henceforth, usually when we refer to an $\omega$-term we will mean its associated word over $Y$. In particular, there is a natural homomorphism of unary semigroups $\epsilon: \mathcal{T}_{X} \rightarrow \Omega_{X}^{\omega} \mathrm{A}$ that fixes each $x \in X$ when we view $X$ as a subset of $\mathcal{T}_{X}$ and $\Omega_{X}^{\omega} \mathrm{A}$ in the natural way. To avoid ambiguities in the meaning of the parentheses, we write $\epsilon[w]$ for the image of $w \in \mathcal{T}_{X}$ under $\epsilon$.

The $\omega$-word problem for A (over $X$ ) consists in deciding whether two given elements of $\mathcal{T}_{X}$ have the same image under $\epsilon$. This problem was solved by McCammond by effectively transforming any $\omega$-term into a certain normal form with the same image under $\epsilon$, and by proving that two $\omega$-terms in normal form with the same image under $\epsilon$ are necessarily equal. In order to describe the normal form, let us fix a total ordering on the alphabet $X$, and extend it to $Y=X \cup\{()$,$\} by letting (<x<)$ for all $x \in X$. The rank of an $\omega$-term $\alpha$ is the maximum number $\operatorname{rank}[\alpha]$ of nested parentheses in it.

McCammond's normal form is defined recursively. Rank-0 normal forms are the words from $X^{*}$. Assuming that rank- $i$ normal forms have been defined, a rank- $(i+1)$ normal form $(\omega$-term) is an $\omega$-term of the form

$$
\alpha_{0}\left(\beta_{1}\right) \alpha_{1}\left(\beta_{2}\right) \ldots \alpha_{n-1}\left(\beta_{n}\right) \alpha_{n}
$$

where the $\alpha_{j}$ and $\beta_{k}$ are $\omega$-terms such that the following conditions hold:

(a) each $\beta_{k}$ is a Lyndon word of rank $i$;

(b) no intermediate $\alpha_{j}$ is a prefix of a power of $\beta_{j}$ or a suffix of a power of $\beta_{j+1}$;

(c) replacing each subterm $\left(\beta_{k}\right)$ by $\beta_{k} \beta_{k}$, we obtain a rank- $i$ normal form;

(d) at least one of the properties (b) and (c) fails if we remove from $\alpha_{j}$ a prefix $\beta_{j}$ (for $0<j$ ) or a suffix $\beta_{j+1}$ (for $j<n$ ).

For instance, if the letters $a, b \in X$ are such that $a<b$, then the terms $(a) a b(b)$, $b(a b) a b a a(a) a a a b(a a b)$ and $((a) a b(b) b a)(a) a b(b)$ are in normal form.

McCammond's procedure to transform an arbitrary $\omega$-term into one in normal form, while retaining its value under $\epsilon$, consists in applying elementary changes determined by the following rewriting rules:

$$
\begin{array}{ll}
\text { 1. }((\alpha)) \rightleftarrows(\alpha) & 4 R .(\alpha) \alpha \rightleftarrows(\alpha) \\
\text { 2. }\left(\alpha^{k}\right) \rightleftarrows(\alpha) & \text { 4L. } \alpha(\alpha) \rightleftarrows(\alpha) \\
\text { 3. }(\alpha)(\alpha) \rightleftarrows(\alpha) & 5 . \quad(\alpha \beta) \alpha \rightleftarrows \alpha(\beta \alpha)
\end{array}
$$

We call the application of a rule of type 1-4 from left to right (respectively, from right to left) a contraction (respectively, an expansion) of that type.

Since all the rules are based on identities of unary semigroups that are valid in A (in fact, all but those of type 4 are valid in S), it follows that the elementary changes preserve the value of the $\omega$-term under $\epsilon$. Hence McCammond's algorithm does indeed transform an arbitrary $\omega$ term into one in normal form with the same image under $\epsilon$. We do not describe McCammond's procedure here because we will usually work with $\omega$-terms already in normal form. The reader interested in the algorithm is referred to the original paper [22], or to [9] for a more condensed description.

\section{Expansions of $\omega$-terms}

The main tool of this paper is to associate to any $\omega$-term $\alpha$ a decreasing sequence $\left(L_{n}[\alpha]\right)_{n}$ of regular languages. Informally, for $n>0$, the language $L_{n}[\alpha]$ is obtained from $\alpha$ by replacing each $\omega$-power by a power of exponent at least $n$. That is, $L_{n}[\alpha]$ is the language obtained from $\alpha$ by replacing each ' $\omega$ ' by ' $\geqslant n$ ', where we set $L^{\geqslant n}=L^{*} L^{n}$ for $L \subseteq X^{+}$. 
Clearly, the sequence $\left(L_{n}[\alpha]\right)_{n}$ is decreasing, and $\epsilon[\alpha]$ belongs to the topological closure, in $\bar{\Omega}_{X} \mathrm{~A}$, of each $L_{n}[\alpha]$. The key result (Theorem 5.1 below) is that $L_{n}[\alpha]$ is star-free for $\alpha$ in normal form and $n$ large enough.

We now formally define $L_{n}[\alpha]$, first defining intermediate expansions that only unfold the outermost $\omega$-powers enclosing subterms of maximum rank. The main differences between this definition and McCammond's 'rank $i$ expansions' [22, Definition 10.5] are that we require the exponents to be beyond a fixed threshold and we do not require that the $\omega$-terms be in normal form.

Definition 1 (Word expansions). Let $n$ be a positive integer. For a word $\alpha \in X^{*}$, we let $E_{n}[\alpha]=\{\alpha\}$. Let $i \geqslant 0$. For an $\omega$-term

$$
\begin{aligned}
\alpha= & \gamma_{0}\left(\delta_{1}\right) \gamma_{1} \ldots\left(\delta_{r}\right) \gamma_{r} \text { where all } \delta_{k} \text { are } \omega \text {-terms of rank } i \text { and all } \gamma_{j} \text { are either } \\
& \text { empty, or } \omega \text {-terms of rank at most } i,
\end{aligned}
$$

we let

$$
E_{n}[\alpha]=\left\{\gamma_{0} \delta_{1}^{n_{1}} \gamma_{1} \ldots \delta_{r}^{n_{r}} \gamma_{r}: n_{1}, \ldots, n_{r} \geqslant n\right\} .
$$

For a set $W$ of $\omega$-terms, we let $E_{n}[W]=\bigcup_{\alpha \in W} E_{n}[\alpha]$. We then let

$$
L_{n}[\alpha]=E_{n}^{\operatorname{rank}[\alpha]}[\alpha],
$$

where $E_{n}^{k}$ is the $k$-fold iteration of the operator $E_{n}$. For a set $W$ of $\omega$-terms, we let $L_{n}[W]=$ $\bigcup_{\alpha \in W} L_{n}[\alpha]$.

For example, let $\alpha=\left(a^{\omega} b\right)^{\omega}$ and $n=3$. We have $\operatorname{rank}[\alpha]=2$, so $L_{3}[\alpha]=E_{3}^{2}[\alpha]$. Then, $E_{3}[\alpha]=\left\{\left(a^{\omega} b\right)^{p} \mid p \geqslant 3\right\}$ and $L_{3}[\alpha]=\left(a^{*} a^{3} b\right)^{*}\left(a^{*} a^{3} b\right)^{3}$.

Lemma 3.1. The following formulas hold:

(a) for $\omega$-terms $\alpha$ and $\beta$,

$$
E_{n}[\alpha \beta]= \begin{cases}E_{n}[\alpha] E_{n}[\beta] & \text { if } \operatorname{rank}[\alpha]=\operatorname{rank}[\beta], \\ \alpha E_{n}[\beta] & \text { if } \operatorname{rank}[\alpha]<\operatorname{rank}[\beta], \\ E_{n}[\alpha] \beta & \text { if } \operatorname{rank}[\alpha]>\operatorname{rank}[\beta] ;\end{cases}
$$

(b) for an $\omega$-term $\alpha, L_{n}[\alpha]=L_{n}\left[E_{n}[\alpha]\right]$;

(c) for sets $U$ and $V$ of $\omega$-terms, we have $L_{n}[U V]=L_{n}[U] L_{n}[V]$;

(d) for a factorization $\alpha=\gamma_{0}\left(\delta_{1}\right) \gamma_{1} \ldots\left(\delta_{r}\right) \gamma_{r}$ of an $\omega$-term as in (3.1),

$$
L_{n}[\alpha]=L_{n}\left[\gamma_{0}\right] L_{n}\left[\left(\delta_{1}\right)\right] L_{n}\left[\gamma_{1}\right] \ldots L_{n}\left[\left(\delta_{r}\right)\right] L_{n}\left[\gamma_{r}\right]
$$

(e) for an $\omega$-term $\alpha, L_{n}[(\alpha)]=L_{n}[\alpha]^{*} L_{n}[\alpha]^{n}$.

Proof. (a) is immediate from the definition of the operator $E_{n}$. For (b), since $E_{n}[\alpha]$ is a set of $\omega$-terms whose rank is $\operatorname{rank}[\alpha]-1$, we have

$$
L_{n}\left[E_{n}[\alpha]\right]=E_{n}^{\operatorname{rank}[\alpha]-1}\left[E_{n}[\alpha]\right]=E_{n}^{\operatorname{rank}[\alpha]}[\alpha]=L_{n}[\alpha] .
$$

We first establish (c) when the rank of elements of $U \cup V$ is bounded by some $m>0$, proceeding by induction on $m$. For $\omega$-terms $\alpha$ and $\beta$ of rank at most $m$, we have

$$
\begin{aligned}
L_{n}[\alpha \beta] \underset{(\mathrm{b})}{=} L_{n}\left[E_{n}[\alpha \beta]\right] & = \begin{cases}L_{n}\left[E_{n}[\alpha] E_{n}[\beta]\right] & \text { if } \operatorname{rank}[\alpha]=\operatorname{rank}[\beta], \\
L_{n}\left[\alpha E_{n}[\beta]\right] & \text { if } \operatorname{rank}[\alpha]<\operatorname{rank}[\beta], \text { by (a) } \\
L_{n}\left[E_{n}[\alpha] \beta\right] & \text { if } \operatorname{rank}[\alpha]>\operatorname{rank}[\beta],\end{cases} \\
& =L_{n}[\alpha] L_{n}[\beta] \text { by induction hypothesis and (b). }
\end{aligned}
$$


To conclude the induction step, note that

$$
L_{n}[U V]=\bigcup_{\alpha \in U, \beta \in V} L_{n}[\alpha \beta]=\bigcup_{\alpha \in U, \beta \in V} L_{n}[\alpha] L_{n}[\beta]=L_{n}[U] L_{n}[V] .
$$

This shows in particular that $L_{n}[\alpha \beta]=L_{n}[\alpha] L_{n}[\beta]$ for all $\omega$-terms $\alpha$ and $\beta$, so that (3.2) still holds for arbitrary sets $U$ and $V$, which establishes (c).

Property (d) follows from (c) by induction on the number of factors. For (e), we have

$$
L_{n}[(\alpha)] \underset{(\mathrm{b})}{=} L_{n}\left[E_{n}[(\alpha)]\right]=\bigcup_{m \geqslant n} L_{n}\left[\alpha^{m}\right] \underset{(\mathrm{c})}{=} \bigcup_{m \geqslant n} L_{n}[\alpha]^{m}=L_{n}[\alpha]^{*} L_{n}[\alpha]^{n} .
$$

If $\alpha$ is a rank- $(i+1) \omega$-term in normal form, the elements of $E_{1}[\alpha]$ are precisely McCammond's 'rank $i$ expansions of $\alpha$ '. Since Lemma 10.7 of [22] states that every such rank- $i$ expansion of $\alpha$ remains in normal form and since $E_{1}[\alpha] \supseteq E_{2}[\alpha] \supseteq E_{3}[\alpha] \supseteq \ldots$, we obtain the following result.

Lemma 3.2. If $\alpha$ is an $\omega$-term in normal form, then all $\omega$-terms of $E_{n}^{k}[\alpha]$ for $n, k \geqslant 1$ are also in normal form.

We now associate to each term $\alpha$ a parameter $\mu[\alpha]$ playing an important role in this paper. First define the length of an $\omega$-term $\alpha$ as the length of the corresponding well-parenthesized word over $Y$, and denote it $|\alpha|$. For an $\omega$-term $\alpha$ as in (3.1), the factors of $\alpha$ of the form $\left(\delta_{j}\right) \gamma_{j}\left(\delta_{j+1}\right)$ are called crucial portions of $\alpha$.

Definition 2. Let $\alpha$ be an $\omega$-term. If $\alpha \in X^{+}$, let $\mu[\alpha]=0$. Otherwise, let

$$
\mu[\alpha]=2^{\operatorname{rank}[\alpha]} \max \left\{|\beta|: \beta \text { is a crucial portion of } \alpha^{2}\right\} .
$$

It is important to point out the following simple observation.

Lemma 3.3. If $\alpha$ is an $\omega$-term and $\bar{\alpha} \in E_{n}[\alpha]$, then $\mu[\bar{\alpha}] \leqslant \mu[\alpha]$.

Proof. The statement is clear if $\operatorname{rank}[\alpha] \leqslant 1$. Otherwise, $\mu[\bar{\alpha}]=2^{\operatorname{rank}[\bar{\alpha}]}|\bar{\beta}|$ for some crucial portion $\bar{\beta}$ of $\bar{\alpha}^{2}$. Since $2^{\operatorname{rank}[\alpha]}=2 \cdot 2^{\operatorname{rank}[\bar{\alpha}]}$, it suffices to show that there exists a crucial portion $\beta$ of $\alpha^{2}$ such that $|\bar{\beta}| \leqslant 2|\beta|$. Since $\bar{\alpha}^{2} \in E_{n}\left(\alpha^{2}\right)$ by Lemma 3.1, $\bar{\beta}$ is a factor of either some $\delta \gamma \delta^{\prime}$ where $(\delta) \gamma\left(\delta^{\prime}\right)$ is a crucial portion of $\alpha^{2}$, or of some $\delta \delta$ where $(\delta)$ is a factor of $\alpha$ of maximum rank. In the first case, choose $\beta=(\delta) \gamma\left(\delta^{\prime}\right)$ so that $|\bar{\beta}| \leqslant|\beta|$. In the second one, take for $\beta$ any crucial portion of $\alpha^{2}$ involving $(\delta)$. Then $|\bar{\beta}| \leqslant 2|\delta| \leqslant 2|\beta|$, as required.

For an $\omega$-term $\alpha$ of positive rank, we distinguish the innermost, rank-1, parentheses as new letters $\llbracket$ and $\rrbracket$. We extend the ordering over the enlarged alphabet $X \cup\{\llbracket, \rrbracket\}$ by letting $\llbracket<x<\rrbracket$ $(x \in X)$. Under this interpretation, we view $\alpha$ as an $\omega$-term over $X \cup\{\llbracket, \rrbracket\}$, denoted $\underline{\alpha}$ and called the freeze of $\alpha$.

REMARK 1. The freeze $\underline{\alpha}$ of an $\omega$-term $\alpha$ satisfies the relations $\operatorname{rank}[\underline{\alpha}]=\operatorname{rank}[\alpha]-1$, and $\mu[\underline{\alpha}] \leqslant \mu[\alpha] / 2$. Moreover, if $\alpha$ (respectively, its crucial portions) is in normal form, then so is $\underline{\alpha}$ (respectively, so are its crucial portions).

\section{A synchronization result}

We prove in this section a synchronization result for $\omega$-terms of rank 1 . 
Proposition 4.1. Let $\alpha=u_{0}\left(v_{1}\right) u_{1} \ldots\left(v_{r}\right) u_{r}$ and $\beta=z_{0}\left(t_{1}\right) z_{1} \ldots\left(t_{s}\right) z_{s}$ be two $\omega$-terms of rank 1 in normal form, and let $n \geqslant \max \{\mu[\alpha], \mu[\beta]\}$. Let

$$
w=u_{0} v_{1}^{n_{1}} u_{1} \ldots v_{r}^{n_{r}} u_{r}=z_{0} t_{1}^{m_{1}} z_{1} \ldots t_{s}^{m_{s}} z_{s} \in L_{n}[\alpha] \cap L_{n}[\beta] .
$$

Then $r=s$, and for all $i, u_{i}=z_{i}, n_{i}=m_{i}$ and $v_{i}=t_{i}$. In particular, $\alpha=\beta$.

The remainder of this section is devoted to the proof of Proposition 4.1. For a factorization $w=u_{0} v_{1}^{n_{1}} u_{1} \ldots v_{r}^{n_{r}} u_{r}$ (which will be clear from the context), we denote by $w[i]$ the word $u_{0} v_{1}^{n_{1}} \ldots u_{i-1} v_{i}^{n_{i}}$; this is empty for $i=0$ by convention.

We shall use the following synchronization property: if two powers of Lyndon words have a large common factor, then the Lyndon words are equal, and the common factor starts in the same position in both of them.

Lemma 4.2. Let $u$ and $v$ be Lyndon words, and let $w$ be a factor of both a power of $u$ and a power of $v: u^{m}=x w y$ and $v^{n}=z w t$. If $|w| \geqslant|u|+|v|$, then $u=v$, and there is a factorization $w=w_{1} w_{2}$ such that $x w_{1}, z w_{1} \in u^{*}$.

Proof. The hypothesis implies that $w$ is a prefix of both a power of a conjugate $\tilde{u}=u_{2} u_{1}$ of $u=u_{1} u_{2}$ and of a power of a conjugate $\tilde{v}=v_{2} v_{1}$ of $v=v_{1} v_{2}$. By Fine and Wilf's theorem (Proposition 2.1) $\tilde{u}$ and $\tilde{v}$ are powers of the same word. Since $\tilde{u}$ and $\tilde{v}$ are primitive, they are equal, hence the Lyndon words in their class, $u$ and $v$ respectively, are also equal.

By symmetry, one may assume that $u_{1} \neq 1$ and $u_{1} \preccurlyeq v_{1}$. Since $u_{2} u_{1}$ and $v_{2} v_{1}$ are conjugates of the same primitive word $u_{1} u_{2}=v_{1} v_{2}$, they are of the form $r s$ and $s r$ with $r=u_{1}^{-1} v_{1}$ and $s=v_{2} u_{1}$. Since they are equal, we obtain $r, s \in p^{*}$ for some word $p$ by [19, Proposition 1.3.2], and since they are primitive, we get $r=1$ or $s=1$, whence $u_{1}=v_{1}$ and $u_{2}=v_{2}$. Moreover, $x=u^{k} u_{1}$ and $z=u^{\ell} v_{1}$. Therefore, $w_{1}=u_{2}=v_{2}$ meets the requirements of the lemma.

REMARK 2. Let $\alpha=u_{0}\left(v_{1}\right) u_{1} \ldots\left(v_{r}\right) u_{r}$ be an $\omega$-term of rank 1 . Let $z$ be a nonempty word, and let $m \geqslant \mu[\alpha]$. Then, for each $i \in\{1, \ldots, r\}$, we have

$$
\left|z^{m}\right| \geqslant\left|u_{i-1} u_{i}\right|+\left|v_{i} z\right|
$$

Indeed, one may assume by symmetry that $\left|u_{i}\right| \geqslant\left|u_{i-1}\right|$. Let $\beta$ be $\left(v_{i}\right) u_{i}\left(v_{i+1}\right)$ if $i<r$, or $\left(v_{r}\right) u_{r} u_{0}\left(v_{1}\right)$ if $i=r$. Since $\beta$ is a crucial portion of $\alpha^{2}$, we have $m \geqslant \mu[\alpha] \geqslant 2|\beta| \geqslant$ $2\left|u_{i}\right|+\left|v_{i}\right|+1 \geqslant\left|u_{i-1} v_{i} u_{i}\right|+1$, so $\left|z^{m}\right| \geqslant\left|z^{\left|u_{i-1} v_{i} u_{i}\right|+1}\right|=\left(\left|u_{i-1} v_{i} u_{i}\right|+1\right)|z| \geqslant\left|u_{i-1} u_{i}\right|+\left|v_{i} z\right|$.

We next consider synchronizations with one single $\omega$-power.

LEMma 4.3. Let $\alpha=u_{0}\left(v_{1}\right) u_{1} \ldots\left(v_{r}\right) u_{r}$ be an $\omega$-term of rank 1 , whose crucial portions are in normal form. Let $z$ be a Lyndon word, and let

$$
w=u_{0} v_{1}^{n_{1}} u_{1} \ldots v_{r}^{n_{r}} u_{r} \in L_{n}[\alpha], \quad \text { with } n \geqslant \max \{\mu[\alpha],|z|+1\} .
$$

Consider a prefix of $w$ of the form $p z^{m}$ with $m \geqslant n$ such that, for some $i \geqslant 1$, the following inequalities hold:

$$
|w[i-1]| \leqslant|p|<|w[i]|
$$

Then $z=v_{i}$ and:

(a) either there is a factorization $u_{i-1}=q v_{i}^{k}$ such that $p=w[i-1] q$;

(b) or there exists $k$ such that $p=w[i-1] u_{i-1} v_{i}^{k}$. 
(a)

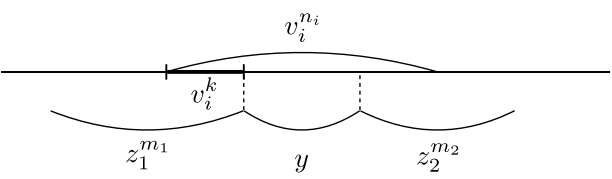

(b)

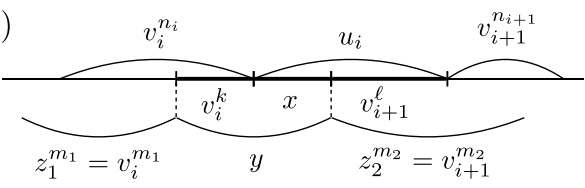

(c)

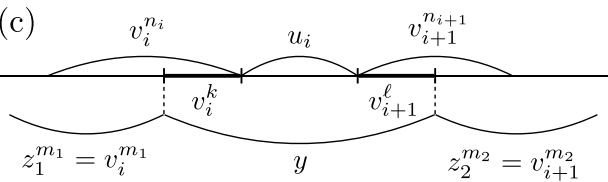

Figure 1. Three factorization patterns when $p z_{1}^{m_{1}} \preccurlyeq w[i]$.

Proof. Let $x=p^{-1} w[i]$. We claim that if $|x| \geqslant\left|v_{i} z\right|$, then $z^{m}$ and $v_{i}^{n_{i}}$ overlap on $\left|v_{i} z\right|$ positions. Suppose first that $|x| \geqslant\left|z^{m}\right|$. Then $z^{m}$ is a prefix of $x$, which in turn is a suffix of $u_{i-1} v_{i}^{n_{i}}$, so indeed $z^{m}$ and $v_{i}^{n_{i}}$ overlap on $\left|z^{m}\right|-\left|u_{i-1}\right| \geqslant\left|v_{i} z\right|$ positions, by (4.1). Consider next the case $\left|v_{i} z\right| \leqslant|x|<\left|z^{m}\right|$. Since

$$
\left|v_{i}^{n_{i}}\right| \geqslant\left|v_{i}^{n}\right| \geqslant\left|v_{i}^{|z|+1}\right|=(|z|+1)\left|v_{i}\right| \geqslant\left|v_{i} z\right|
$$

one can consider the suffix $u$ of $v_{i}^{n_{i}}$ of length $\left|v_{i} z\right|$. Since $x$ and $v_{i}^{n_{i}}$ are suffixes of the same word and $|x| \geqslant\left|v_{i} z\right|, u$ is a suffix of $x$. Since $x$ is a prefix of $z^{m}, u$ is a factor of $z^{m}$. This proves the claim, so by Lemma 4.2 applied to $v_{i}^{n_{i}}$ and $z^{m}$, we conclude that $z=v_{i}$, and that (a) or (b) holds, depending on whether or not we have $|p|<\left|w[i-1] u_{i-1}\right|$.

Finally, assume that $|x|<\left|v_{i} z\right|$. From (4.1), we get $\left|z^{m}\right|>\left|x u_{i}\right|$ and so $i<r$. Hence, using $m \geqslant \mu[\alpha] \geqslant 2\left|v_{i} u_{i} v_{i+1}\right| \geqslant\left|v_{i} u_{i} v_{i+1}\right|+2$,

$$
\left|z^{m}\right|-\left|x u_{i}\right| \geqslant\left|z^{\left|v_{i} u_{i} v_{i+1}\right|+2}\right|-\left|x u_{i}\right| \geqslant\left|v_{i} u_{i} v_{i+1}\right|+2|z|-\left|x u_{i}\right|>\left|v_{i+1} z\right| .
$$

Therefore, $z^{m}$ and $v_{i+1}^{n_{i+1}}$ have a common factor of length at least $\left|v_{i+1} z\right|$. By Lemma 4.2 again, we have $z=v_{i+1}$ and $p z^{k}=w[i] u_{i}$ for some $k$ such that $1 \leqslant k<m$. Since $|p|<|w[i]|$, it follows that $u_{i}$ is a suffix of $z^{k}=v_{i+1}^{k}$, contradicting the hypothesis that $v_{i}^{\omega} u_{i} v_{i+1}^{\omega}$ is a crucial portion in normal form. This concludes the proof of the lemma.

We now develop the inductive argument in order to prove Proposition 4.1.

LEMma 4.4. Let $\alpha=u_{0}\left(v_{1}\right) u_{1} \ldots\left(v_{r}\right) u_{r}$ and $\beta=\left(z_{1}\right) y\left(z_{2}\right)$ be $\omega$-terms of rank 1 whose crucial portions are in normal form. Let

$$
w=u_{0} v_{1}^{n_{1}} u_{1} \ldots v_{r}^{n_{r}} u_{r} \in L_{n}[\alpha], \quad \text { with } n \geqslant \max \{\mu[\alpha], \mu[\beta]\} .
$$

If there is a prefix of $w$ of the form $p z_{1}^{m_{1}} y z_{2}^{m_{2}}$ with $m_{1}, m_{2} \geqslant n$ and

$$
|w[i-1]| \leqslant|p|<|w[i]|,
$$

then $z_{1}=v_{i}, i<r, y=u_{i}, z_{2}=v_{i+1}$, and $p z_{1}^{m_{1}}=w[i]$.

Proof. Lemma 4.3 shows that $z_{1}=v_{i}$. We first assume that $p z_{1}^{m_{1}} \preccurlyeq w[i]$. If $p z_{1}^{m_{1}} y \preccurlyeq w[i]$ (case (a) of Figure 1), then $y$ would be a prefix of a power of $v_{i}=z_{1}$, which is impossible since $\beta$ is in normal form. Hence $p z_{1}^{m_{1}} \preccurlyeq w[i] \prec p z_{1}^{m_{1}} y$, so $y$ and $u_{i}$ overlap. Consider the cases $p z_{1}^{m_{1}} y \preccurlyeq w[i] u_{i}$ and $p z_{1}^{m_{1}} y \succ w[i] u_{i}$ (cases (b) and (c) of Figure 1, in which the references to $v_{i+1}$ underneath the straight line are justified below). We claim that $i>r$ and that $z_{2}^{m_{2}}$ and $v_{i+1}^{n_{i+1}}$ overlap on $\left|v_{i+1} z_{2}\right|$ positions in $w$. In case (b), $z_{2}^{m_{2}}>\left|u_{i}\right|$ by (4.1) applied to $z=z_{2}$, 

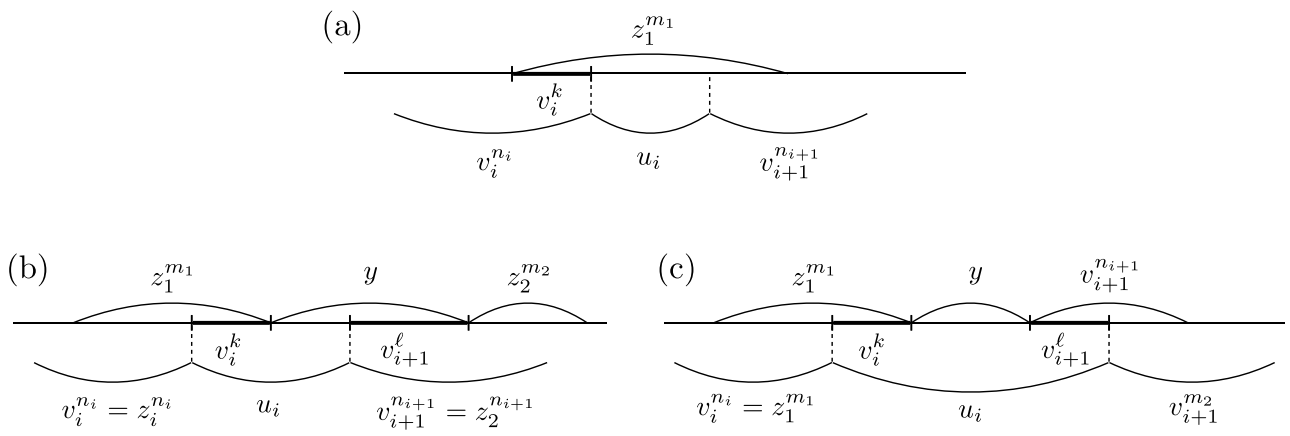

FigURE 2. Three factorization patterns when $p z_{1}^{m_{1}} \succcurlyeq w[i]$.

hence $i<r$, and (4.1) applied at index $i+1$ instead of $i$ yields $\left|z_{2}^{m_{2}}\right| \geqslant\left|u_{i}\right|+\left|v_{i+1} z_{2}\right|$, so $z_{2}^{m_{2}}$ and $v_{i+1}^{n_{i+1}}$ overlap on $\left|v_{i+1} z_{2}\right|$ positions in $w$. In case (c), $i>r$ is clear from the assumption that $p z_{1}^{m_{1}} y \succ w[i] u_{i}$. Finally, we obtain $\left|v_{i+1}^{n_{i+1}}\right|=n_{i+1}\left|v_{i+1}\right| \geqslant \mu[\beta]\left|v_{i+1}\right| \geqslant 2\left|z_{1} y z_{2}\right|\left|v_{i+1}\right|$, whence $\left|v_{i+1}^{n_{i+1}}\right|-|y| \geqslant\left|z_{2} v_{i+1}\right|$. Similarly, we have $\left|z_{2}^{m_{2}}\right| \geqslant \mu[\alpha]\left|z_{2}\right| \geqslant\left|z_{2} v_{i+1}\right|$, so $z_{2}^{m_{2}}$ and $v_{i+1}^{n_{i+1}}$ overlap on $\left|v_{i+1} z_{2}\right|$ positions.

Therefore $z_{2}=v_{i+1}$ by Lemma 4.2 and for some $k, \ell \geqslant 0$, we have $y=v_{i}^{k} x$ and $u_{i}=x v_{i+1}^{\ell}$ in case (b), where $x$ is the overlap between $y$ and $u_{i}$, and $y=v_{i}^{k} u_{i} v_{i+1}^{\ell}$ in case (c). We claim that in either case, $k=\ell=0$, which proves the statement. In case (b), $x$ is not a prefix of a power of $z_{1}$ since, otherwise, so would be $y$, contradicting the fact that $\beta$ is in normal form. On the other hand, $x$ is not a suffix of a power of $z_{2}$ since, otherwise, so would be $u_{i}$, contradicting the fact that $\left(v_{i}\right) u_{i}\left(v_{i+1}\right)$ is in normal form. Therefore, $\left(v_{i}\right) x\left(v_{i+1}\right)$ is in normal form. Since $\left(v_{i}\right) u_{i}\left(v_{i+1}\right)$ is also in normal form, we deduce in both cases, by condition (d) of the definition of normal form, that $k=\ell=0$.

Finally, assume that $p z_{1}^{m_{1}} \succcurlyeq w[i]$. The resulting three factorization patterns are depicted in Figure 2. Note that they are in correspondence with the factorization patterns in Figure 1. The arguments presented above for the case $p z_{1}^{m_{1}} \preccurlyeq w[i]$ therefore apply, mutatis mutandis, to the current case.

Proof of Proposition 4.1. We have $\left|v_{1}^{n_{1}}\right| \geqslant\left|v_{1}\right|+n_{1}-1 \geqslant\left|v_{1}\right|+2\left|z_{0} t_{1}\right|-1 \geqslant\left|z_{0} t_{1} v_{1}\right|$. Likewise, $\left|t_{1}^{m_{1}}\right| \geqslant\left|u_{0} v_{1} t_{1}\right|$, so $v_{1}^{n_{1}}$ and $t_{1}^{m_{1}}$ overlap on a factor of length at least $\left|v_{1} t_{1}\right|$. By Lemma 4.2, $v_{1}=t_{1}$ and $z_{0}=u_{0} t_{1}^{k}$ or $u_{0}=z_{0} v_{1}^{k}$ for some $k$. Since $\alpha$ and $\beta$ are in normal form, $k=0$ by property (d) of normal forms. Hence $u_{0}=z_{0}$. Suppose inductively that for $i \geqslant 1$, we have $u_{k-1}=z_{k-1}, n_{k-1}=m_{k-1}$ and $v_{k}=t_{k}$ for all $1 \leqslant k \leqslant i$. If $i<s$, then one can apply Lemma 4.4 , since the portion $\left(t_{i}\right) z_{i}\left(t_{i+1}\right)$ is in normal form and $w[i-1] \preccurlyeq z_{0} t_{1}^{m_{1}} z_{1} \ldots t_{i-1}^{m_{i-1}} z_{i-1} \prec w[i]$, where the notation $w[\cdot]$ refers to the first factorization, so that (4.2) is fulfilled for the word $p=z_{0} t_{1}^{m_{1}} z_{1} \ldots t_{i-1}^{m_{i}-1} z_{i-1}$. This yields $i<r, u_{i}=z_{i}$, $n_{i}=m_{i}$ and $v_{i+1}=t_{i+1}$. The case $i<r$ is dual. Finally, if $i=r=s$, then we obtain $u_{r}=z_{r}$ by left-right symmetry, so $n_{r}=m_{r}$.

\section{The $\omega$-word problem over A}

In this section we reveal how the languages $L_{n}[\alpha]$ can be used to obtain an alternative proof of McCammond's solution of the word problem for $\omega$-terms over A. The fundamental property of the languages $L_{n}[\alpha]$, whose proof is presented in the next section, is their star-freeness under suitable hypotheses. 
Theorem 5.1. Let $\alpha$ be an $\omega$-term in normal form and let $n \geqslant \mu[\alpha]$. Then the language $L_{n}[\alpha]$ is star-free.

A simpler but also important property is stated in Lemma 5.2 below, which follows from the synchronization property of Proposition 4.1. For an $\omega$-term $\alpha$, we set $E_{n}^{*}[\alpha]=\bigcup_{i \geqslant 0} E_{n}^{i}[\alpha]$.

Lemma 5.2. Let $\alpha$ and $\beta$ be two $\omega$-terms in normal form with $\operatorname{rank}[\beta] \geqslant \operatorname{rank}[\alpha]$, and let $n>\max \{\mu[\alpha], \mu[\beta]\}$. If $L_{n}[\alpha] \cap L_{n}[\beta] \neq \emptyset$, then $\alpha \in E_{n}^{*}[\beta]$.

Proof. Let $w \in L_{n}[\alpha] \cap L_{n}[\beta]$. We proceed by induction on $\operatorname{rank}[\alpha]=i$. If $i=0$, that is, $\alpha \in X^{+}$, we have $w=\alpha$ so that $\alpha \in L_{n}[\beta]=E_{n}^{\operatorname{rank}[\beta]}[\beta]$. Assume next that $i \geqslant 1$ and that the result holds for $\operatorname{rank}[\alpha]<i$. By definition of $L_{n}$ and the choice of $w$, there exist $\alpha_{1} \in E_{n}^{\operatorname{rank}[\alpha]-1}[\alpha]$ and $\beta_{1} \in E_{n}^{\operatorname{rank}[\beta]-1}[\beta]$ such that $w \in L_{n}\left[\alpha_{1}\right] \cap L_{n}\left[\beta_{1}\right]$. By Lemma 3.2, the $\omega$-terms $\alpha_{1}$ and $\beta_{1}$ are in normal form. Let $u_{0}\left(v_{1}\right) u_{1} \ldots\left(v_{r}\right) u_{r}$ and $z_{0}\left(t_{1}\right) z_{1} \ldots\left(t_{s}\right) z_{s}$ be the normal-form expressions of $\alpha_{1}$ and $\beta_{1}$, respectively. We have $n \geqslant \max \left\{\mu\left[\alpha_{1}\right], \mu\left[\beta_{1}\right]\right\}$ by Lemma 3.3. By Proposition 4.1, it follows that $\alpha_{1}=\beta_{1}$, so

$$
E_{n}^{\operatorname{rank}[\alpha]-1}[\alpha] \cap E_{n}^{\operatorname{rank}[\beta]-1}[\beta] \neq \emptyset .
$$

If $i=1$, then $\alpha_{1}=\alpha$ so that $\alpha \in E_{n}^{\operatorname{rank}[\beta]-1}[\beta]$. If $i>1$, consider the freezes $\underline{\alpha}$ and $\beta$. Then $L_{n}[\underline{\alpha}] \cap L_{n}[\underline{\beta}] \neq \emptyset$ follows from (5.1), and by Remark 1, $\underline{\alpha}$ and $\underline{\beta}$ are in normal form, $n>\max \{\mu[\underline{\alpha}], \mu[\overline{\beta]}\}$, and $\operatorname{rank}[\beta] \geqslant \operatorname{rank}[\underline{\alpha}]=i-1$. By the induction hypothesis, we obtain $\underline{\alpha} \in E_{n}^{*}[\beta]$ and, therefore, $\alpha \in E_{n}^{*}[\beta]$, which completes the induction step and the proof of the lemma.

By raising the lower bound for $n$, we obtain a more precise result.

TheOREM 5.3. Let $\alpha$ and $\beta$ be two $\omega$-terms in normal form and let $n$ be an integer such that $n>\max \{|\alpha|,|\beta|, \mu[\alpha], \mu[\beta]\}$. If $L_{n}[\alpha] \cap L_{n}[\beta] \neq \emptyset$, then $\alpha=\beta$.

Proof. Suppose that $\operatorname{rank}[\alpha] \leqslant \operatorname{rank}[\beta]$, so that, by Lemma $5.2, \alpha \in E_{n}^{*}[\beta]$. If $\operatorname{rank}[\beta]>$ $\operatorname{rank}[\alpha]$, it follows that $|\alpha| \geqslant n$, which contradicts the assumption on $n$. Hence we must have $\operatorname{rank}[\beta]=\operatorname{rank}[\alpha]$ and so $\alpha=\beta$.

Combining Theorems 5.1 and 5.3, we obtain a new proof of uniqueness of McCammond's normal form for elements of $\Omega_{X}^{\omega}$ A.

Corollary 5.4 (McCammond's solution of the $\omega$-word problem over A [22]). Let $\alpha$ and $\beta$ be $\omega$-terms in normal form which define the same pseudoword over $\mathrm{A}$, that is, such that $\epsilon[\alpha]=\epsilon[\beta]$. Then $\alpha=\beta$.

Proof. Let $n>\max \{|\alpha|,|\beta|, \mu[\alpha], \mu[\beta]\}$. Since $L_{n}[\alpha]$ and $L_{n}[\beta]$ are star-free languages by Theorem 5.1, their respective closures $\operatorname{cl}_{\mathrm{A}}\left(L_{n}[\alpha]\right)$ and $\operatorname{cl}_{\mathrm{A}}\left(L_{n}[\beta]\right)$ in $\bar{\Omega}_{X} \mathrm{~A}$ are clopen subsets. Since $\epsilon[\alpha]=\epsilon[\beta] \in \operatorname{cl}_{\mathrm{A}}\left(L_{n}[\alpha]\right) \cap \operatorname{cl}_{\mathrm{A}}\left(L_{n}[\beta]\right)$, the nonempty open set $\operatorname{cl}_{\mathrm{A}}\left(L_{n}[\alpha]\right) \cap \operatorname{cl}_{\mathrm{A}}\left(L_{n}[\beta]\right)$ contains some elements of the dense set $X^{+}$, which in turn belong to $L_{n}[\alpha] \cap L_{n}[\beta]$ since, by $\left[\mathbf{5}\right.$, Theorem 3.6], we have $\operatorname{cl}_{\mathrm{A}}\left(L_{n}[\gamma]\right) \cap X^{+}=L_{n}[\gamma](\gamma \in\{\alpha, \beta\})$. Therefore, $L_{n}[\alpha] \cap L_{n}[\beta] \neq \emptyset$, whence $\alpha=\beta$ by Theorem 5.3.

\section{Star-freeness of the languages $L_{n}[\alpha]$}

This section is dedicated to the proof of Theorem 5.1. 
We say that an $\omega$-term $\alpha$ is in circular normal form if the crucial portions of $\alpha^{2}$ are in normal form. A consequence of Lemma 3.2 is that the property of being in circular normal form is preserved by expansions.

Lemma 6.1. Let $\alpha$ be an $\omega$-term in circular normal form and let $\beta \in E_{n}[\alpha]$. Then $\beta$ is also in circular normal form.

Proof. If $\alpha$ is a word, then $\beta=\alpha$ is certainly in circular normal form. Otherwise $\alpha$ and $\beta$ are of the form $\alpha=\gamma_{0}\left(\delta_{1}\right) \gamma_{1} \ldots\left(\delta_{r}\right) \gamma_{r}$ and $\beta=\gamma_{0} \delta_{1}^{n_{1}} \gamma_{1} \ldots \delta_{r}^{n_{r}} \gamma_{r}$ with each $n_{k} \geqslant n$. Now $\beta^{2} \in E_{n}[\alpha]^{2}=E_{n}\left[\alpha^{2}\right]$ according to Lemma 3.1(a). By Lemma 3.2, applied to the crucial portions of $\alpha^{2}$ which are by hypothesis in normal form, we conclude that all factors $\delta_{k}^{n_{k}} \gamma_{k} \delta_{k+1}^{n_{k+1}}$, as well as $\delta_{r}^{n_{r}} \gamma_{r} \gamma_{0} \delta_{1}^{n_{1}}$, are in normal form. Since each crucial portion of $\beta^{2}$ is a crucial portion of one of these factors, it is in normal form, hence $\beta$ is in circular normal form.

Let us now derive a corollary of Proposition 4.1, which applies to $\omega$-terms in circular normal form (rather than to $\omega$-terms in normal form as in the proposition).

Corollary 6.2 (Proposition 4.1). Let $\alpha=\left(v_{1}\right) u_{1} \ldots\left(v_{r}\right) u_{r}$ and $\beta=\left(t_{1}\right) z_{1} \ldots\left(t_{s}\right) z_{s}$ be two $\omega$-terms of rank 1 in circular normal form, and let $n \geqslant \max \{\mu[\alpha], \mu[\beta]\}$. If $L_{n}[\alpha] \cap L_{n}[\beta] \neq \emptyset$, then $\alpha=\beta$.

Proof. Let $w=v_{1}^{n_{1}} u_{1} \ldots v_{r}^{n_{r}} u_{r}=t_{1}^{m_{1}} z_{1} \ldots t_{s}^{m_{s}} z_{s} \in L_{n}[\alpha] \cap L_{n}[\beta]$ and let $\alpha^{\prime}$ and $\beta^{\prime}$ be the normal forms of $\alpha$ and $\beta$, respectively. As $\alpha$ is in circular normal form by hypothesis, all its crucial portions are in normal form. Therefore $\alpha^{\prime}$ is obtained from $\alpha$ by simply reducing the final portion $\left(v_{r}\right) u_{r}$ to its normal form. This is done by applying all possible, say $k \geqslant 0$, reductions of type $4 R$. That is, $\alpha^{\prime}=\left(v_{1}\right) u_{1} \ldots\left(v_{r}\right) u_{r}^{\prime}$ with $u_{r}=v_{r}^{k} u_{r}^{\prime}$. Analogously, $\beta^{\prime}=\left(t_{1}\right) z_{1} \ldots\left(t_{s}\right) z_{s}^{\prime}$ with $z_{s}=t_{s}^{\ell} z_{s}^{\prime}$ for some $\ell \geqslant 0$. Clearly $\mu[\alpha] \geqslant \mu\left[\alpha^{\prime}\right]$ and $\mu[\beta] \geqslant \mu\left[\beta^{\prime}\right]$, whence $n \geqslant \max \left\{\mu\left[\alpha^{\prime}\right], \mu\left[\beta^{\prime}\right]\right\}$. On the other hand, $w=v_{1}^{n_{1}} u_{1} \ldots v_{r}^{n_{r}+k} u_{r}^{\prime}=t_{1}^{m_{1}} z_{1} \ldots t_{s}^{m_{s}+\ell} z_{s}^{\prime}$ belongs to $L_{n}\left[\alpha^{\prime}\right] \cap L_{n}\left[\beta^{\prime}\right]$. Hence, $\alpha^{\prime}=\beta^{\prime}$ by Proposition 4.1. In particular, $v_{1}=t_{1}, v_{r}=t_{s}$ and $u_{r}^{\prime}=z_{s}^{\prime}$. The crucial portions $\left(v_{r}\right) u_{r}\left(v_{1}\right)$ and $\left(t_{s}\right) z_{s}\left(t_{1}\right)$ of $\alpha^{2}$ and $\beta^{2}$ respectively are in normal form. Then, as $\left(v_{r}\right) u_{r}\left(v_{1}\right)=\left(v_{r}\right) v_{r}^{k} u_{r}^{\prime}\left(v_{1}\right)$ and $\left(t_{s}\right) z_{s}\left(t_{1}\right)=\left(v_{r}\right) v_{r}^{\ell} u_{r}^{\prime}\left(v_{1}\right)$, we deduce from property (d) of normal forms that $k=\ell$. This completes the proof that $\alpha=\beta$.

The next lemma reflects periodicities of sufficiently large expansions of an $\omega$-term of rank 1 in the term itself, provided it is in circular normal form.

Lemma 6.3. Let $\alpha$ be an $\omega$-term of rank 1 in circular normal form and let $n \geqslant \mu[\alpha]$. If $z^{\ell} \in L_{n}[\alpha]$, then there exists an $\omega$-term of rank 1 in circular normal form $\zeta$ such that $\alpha=\zeta^{\ell}$ and $z \in L_{n}[\zeta]$.

Proof. Let $\alpha=u_{0}\left(v_{1}\right) u_{1} \ldots\left(v_{r}\right) u_{r}$. Since $z^{\ell} \in L_{n}[\alpha]$, either $z \prec u_{0} \prec z^{\ell}$ or $u_{0} \preccurlyeq z$. In both cases, we reduce the question to the case where $u_{0}$ is empty, by replacing $\alpha$ by $\left(v_{1}\right) u_{1} \ldots\left(v_{r}\right) u_{r} u_{0}$ and $z$ by an appropriate conjugate, $z_{1}^{-1} z z_{1}$, where $z=z_{1} z_{2}$ and $u_{0}=\left(z_{1} z_{2}\right)^{k} z_{1}$ in the first case, or $u_{0}^{-1} z u_{0}$ in the second case. So write $\alpha=\left(v_{1}\right) u_{1} \ldots\left(v_{r}\right) u_{r}$, and let $w=z^{\ell}$. Then we have $w=v_{1}^{n_{1}} u_{1} \ldots v_{r}^{n_{r}} u_{r}$, with $n_{1}, \ldots, n_{r} \geqslant n$. Taking into account the resulting factorization of $w^{2}$, we also set $v_{r+i}=v_{i}, u_{r+i}=u_{i}$, and $n_{r+i}=n_{i}$ for $i=1, \ldots, r$. Note also that $w^{2}[i+r]=w w[i]$ for $1 \leqslant i \leqslant r$.

If $\ell=1$, then we choose $\zeta=\alpha$. For $\ell \geqslant 2$, assume first that $|z| \leqslant\left|v_{1}^{n_{1}}\right|$. Since both $z$ and $v_{1}^{n_{1}}$ are prefixes of $w$, this implies that $v_{1}^{k-1} \prec z \preccurlyeq v_{1}^{k}$ for some $k \geqslant 1$. Then $t=z^{-1} v_{1}^{k}$ is a suffix of $\left(v_{1}^{k-1}\right)^{-1} v_{1}^{k}=v_{1}$. Further, $v_{1} \preccurlyeq w$ and $t \preccurlyeq z^{-1} w=z^{\ell-1} \prec z^{\ell}=w$, so $t \preccurlyeq v_{1}$. Since $t$ is both a prefix and a suffix of the Lyndon word $v_{1}, t$ is either empty or equal to $v_{1}$ by Lemma 2.2, 
hence $z=v_{1}^{k}$ and $w=z^{\ell}=v_{1}^{k \ell}$. It follows that $u_{1} \preccurlyeq v_{1}^{k \ell-n_{1}}$, which contradicts the hypothesis on $\alpha$. Therefore, we have $\left|v_{1}^{n_{1}}\right|<|z|$ and $v_{1}^{n_{1}} \prec z$.

In particular, the equalities $|z|>n_{1} \geqslant n \geqslant \mu[\alpha]>\left|u_{r}\right|$ hold, so that $w[1] \prec z \preccurlyeq z^{\ell-1} \prec w[r]$. Hence $r \geqslant 2$ and there exists $i \in\{1, \ldots, r-1\}$ such that $w[i] \preccurlyeq z \prec w[i+1]$, which is the same as $w^{2}[i] \preccurlyeq z \prec w^{2}[i+1]$. We prove the following property by induction on $k \in\{1, \ldots, r\}$ :

$$
\begin{cases}z w[j]=w^{2}[i+j] \quad \text { and } \quad u_{j}=u_{i+j} & \text { for } j \leqslant k, \\ v_{j}=v_{i+j} & \text { for } j \leqslant k+1 .\end{cases}
$$

Observe that $z w v_{1}^{n_{1}} \preccurlyeq z^{\ell+2} \preccurlyeq z^{2 \ell}=w^{2}$. We will apply Lemma 4.4 to $\alpha^{2}$ several times, choosing prefixes of $z w v_{1}^{n_{1}}$ for the successive values of the prefix $p$ of $w^{2} \in L_{n}\left[\alpha^{2}\right]$ which is considered in that lemma. First, since $w^{2}[i] \preccurlyeq z \prec w^{2}[i+1]$ and $n \geqslant \mu[\alpha]=\mu\left[\alpha^{2}\right]$, we may apply Lemma 4.4 to $\alpha^{2}$, with $\beta=\left(v_{1}\right) u_{1}\left(v_{2}\right)$ and $p=z$, to obtain $z w[1]=z v_{1}^{n_{1}}=w^{2}[i+1], v_{1}=v_{i+1}, u_{1}=u_{i+1}$, and $v_{2}=v_{i+2}$, which establishes $(H(1))$. Next, assuming that $(H(k-1))$ holds for a certain $k \leqslant r$, we deduce that $w^{2}[i+k-1] \preccurlyeq z w[k-1] u_{k-1} \prec w^{2}[i+k]$. Lemma 4.4 applied to $\alpha^{2}$ with $\beta=\left(v_{k}\right) u_{k}\left(v_{k+1}\right)$ and $p=z w[k-1] u_{k-1}$ then yields $(H(k))$.

In particular, $z w[r]=w^{2}[i+r]=w w[i]$ and $u_{r}=u_{i+r}=u_{i}$. It follows that $z w=z w[r] u_{r}=$ $w w[i] u_{i}$. Since $z w=w z\left(=z^{\ell+1}\right)$, we deduce that $z=w[i] u_{i}=v_{1}^{n_{1}} u_{1} \ldots v_{i}^{n_{i}} u_{i}$. Let $\zeta=$ $\left(v_{1}\right) u_{1} \ldots\left(v_{i}\right) u_{i}$. Then $z$ belongs to $L_{n}[\zeta]$ and $z^{\ell} \in L_{n}\left[\zeta^{\ell}\right] \cap L_{n}[\alpha]$. Since each crucial portion of $\zeta^{2}$ is a crucial portion of $\alpha^{2}$, we have $\mu\left[\zeta^{\ell}\right]=\mu[\zeta] \leqslant \mu[\alpha] \leqslant n$. Therefore, $\alpha=\zeta^{\ell}$ by Corollary 6.2, which completes the proof.

We call primitive an $\omega$-term which is primitive when represented as a parenthesized word. An immediate consequence of Lemma 6.3 is the following observation.

Corollary 6.4. Let $\alpha$ be an $\omega$-term of rank 1 in circular normal form and let $n \geqslant \mu[\alpha]$. If $\alpha$ is primitive and $w \in L_{n}[\alpha]$, then $w$ is also primitive.

The next result may be regarded as a generalization of Corollary 6.4 to $\omega$-terms of larger rank.

Corollary 6.5. Let $\alpha$ be an $\omega$-term of rank $i \geqslant 1$ in circular normal form and let $n \geqslant \mu[\alpha]$. If $\alpha$ is primitive and $\beta \in E_{n}[\alpha]$, then $\beta$ is also primitive.

Proof. We distinguish two types of parentheses in the $\omega$-term $\alpha$ : write (, ) for the parentheses corresponding to the $\omega$-powers of rank $i$, and $\llbracket$, for the remaining parentheses. Consider the alphabet $Z=X \cup\{\llbracket, \rrbracket\}$, with the extended ordering $\llbracket<x<\rrbracket(x \in X)$. Then $\beta$ may be viewed as a word $\beta_{Z}$ over $Z$ and $\alpha$ as an $\omega$-term $\alpha_{Z}$, of rank 1, over the same alphabet such that $\beta_{Z} \in L_{n}\left[\alpha_{Z}\right]$. Moreover, $\mu\left[\alpha_{Z}\right] \leqslant \mu[\alpha]$ and it is clear by McCammond's definition of rank- $i$ normal form that $\alpha_{Z}$ is a primitive $\omega$-term in circular normal form (over $Z$ ), whence $\alpha_{Z}$ and $\beta_{Z}$ satisfy the hypotheses of Corollary 6.4. To conclude the proof, it suffices to invoke Corollary 6.4.

Iterating the application of Corollary 6.5, we obtain another extension of Corollary 6.4 to $\omega$-terms of any rank.

Proposition 6.6. Let $\alpha$ be an $\omega$-term in circular normal form and let $n \geqslant \mu[\alpha]$. If $\alpha$ is a primitive $\omega$-term and $w \in L_{n}[\alpha]$, then $w$ is a primitive word.

Proof. We proceed by induction on $\operatorname{rank}[\alpha]$. The case $\operatorname{rank}[\alpha]=1$ is given by Corollary 6.4. Assume that the result holds for $\omega$-terms whose $\operatorname{rank}$ is $\operatorname{rank}[\alpha]-1 \geqslant 1$. By definition of $L_{n}[\alpha]$, there is an $\omega$-term $\alpha^{\prime} \in E_{n}[\alpha]$ such that $w \in L_{n}\left[\alpha^{\prime}\right]$. By Corollary 6.5, $\alpha^{\prime}$ is primitive. Moreover, $\mu\left[\alpha^{\prime}\right] \leqslant \mu[\alpha]$ by Lemma 3.3, and $\alpha^{\prime}$ is in circular normal form by Lemma 6.1. Hence, by induction hypothesis, $w$ is primitive, which completes the induction step. 


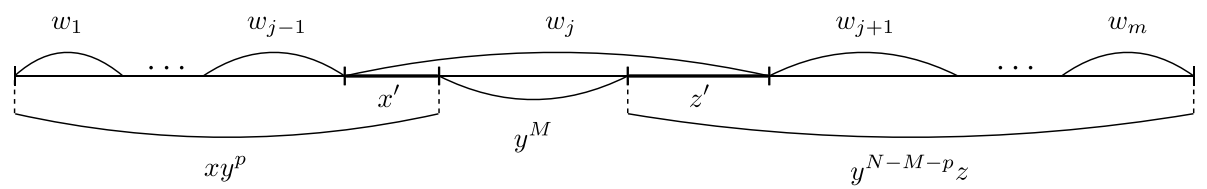

FiguRE 3. The case where some $y^{M}$ falls within some $w_{j}$.

The following result generalizes Lemma 6.3 in the case where $\alpha$ is a primitive $\omega$-term.

Lemma 6.7. Let $\alpha$ be a primitive $\omega$-term in circular normal form and let $n \geqslant \mu[\alpha]$. If $z^{\ell} \in L_{n}[\alpha]^{k}$ then $z \in L_{n}[\alpha]^{m}$ for some $m$ such that $1 \leqslant m \leqslant k$.

Proof. We proceed by induction on $\operatorname{rank}[\alpha]$. If $\operatorname{rank}[\alpha]=0$, then $\alpha$ is a word, so $L_{n}[\alpha]=\{\alpha\}$ and $z^{\ell}=\alpha^{k}$. By [19, Proposition 1.3.1], $z$ and $\alpha$ are powers of the same word, whence $z=\alpha^{m}$ since $\alpha$ is primitive, and $m=k / \ell \leqslant k$. Assume now that $\operatorname{rank}[\alpha] \geqslant 1$ and that the result holds at lower ranks.

Since $L_{n}[\alpha]^{k}=L_{n}\left[\alpha^{k}\right]$ by Lemma 3.1(c), we have $z^{\ell} \in E_{n}\left[E_{n}^{\operatorname{rank}[\alpha]-1}\left[\alpha^{k}\right]\right]$. Pick an $\omega$-term $\beta \in E_{n}^{\operatorname{rank}[\alpha]-1}\left[\alpha^{k}\right]$ of rank 1 such that $z^{\ell} \in E_{n}[\beta]=L_{n}[\beta]$. Since $\alpha$ is in circular normal form, so is $\alpha^{k}$, whence so is $\beta$ by Lemma 6.1 . Since $n \geqslant \mu[\alpha]=\mu\left[\alpha^{k}\right] \geqslant \mu[\beta]$ by Lemma 3.3, one can apply Lemma 6.3: there exists an $\omega$-term $\zeta$ of rank 1 such that $\beta=\zeta^{\ell}$ and $z \in L_{n}[\zeta]$.

If $\operatorname{rank}[\alpha]=1$, then $\zeta^{\ell} \in E_{n}^{\operatorname{rank}[\alpha]-1}\left[\alpha^{k}\right]=\left\{\alpha^{k}\right\}$. Since $\alpha$ is primitive, it follows that $\zeta=\alpha^{m}$, for some $m \leqslant k$, and $z \in L_{n}[\zeta]=L_{n}[\alpha]^{m}$, as required. If $\operatorname{rank}[\alpha]>1$, let us check that we may apply the induction hypothesis to the freeze $\underline{\alpha}$ of $\alpha$ and $\underline{\zeta} \in(X \cup\{\llbracket, \rrbracket\})^{*}$. First, $\underline{\zeta}^{\ell} \in L_{n}\left[\underline{\alpha}^{k}\right]$. Next, since the crucial portions of $\alpha^{2}$ are in normal form, so are those of $\underline{\alpha \cdot \alpha}=\underline{\alpha} \cdot \underline{\alpha}$ by Remark 1 , whence $\underline{\alpha}$ is in circular normal form. Finally, the relations $n \geqslant \mu[\alpha] \geqslant \mu[\underline{\alpha}]$ and $\operatorname{rank}[\underline{\alpha}]=\operatorname{rank}[\alpha]-1$ hold, also by Remark 1. By induction, we therefore obtain $m$ such that $1 \leqslant m \leqslant k$ and $\underline{\zeta} \in L_{n}\left[\underline{\alpha}^{m}\right]$. We deduce that $\zeta \in E_{n}^{\operatorname{rank}[\alpha]-1}\left[\alpha^{m}\right]$, so that $z \in L_{n}[\zeta] \subseteq$ $E_{n}^{\operatorname{rank}[\alpha]}\left[\alpha^{m}\right]=L_{n}\left[\alpha^{m}\right]$.

We proceed to establish the following important property of the languages $L_{n}[\alpha]$ for primitive $\omega$-terms $\alpha$. In its proof, we apply in both directions Schützenberger's theorem [27], stating that a language is star-free if and only if its syntactic semigroup is finite and satisfies the pseudoidentity $x^{\omega+1}=x^{\omega}$.

Lemma 6.8. Let $\alpha$ be a primitive $\omega$-term in circular normal form and let $n \geqslant \mu[\alpha]$. If $L_{n}[\alpha]$ is a star-free language, then so is $L_{n}[\alpha]^{*}$.

Proof. Let $M$ be an integer such that the syntactic semigroup of $L_{n}[\alpha]$ satisfies the identity $x^{M}=x^{M+1}$ and let $K$ be a positive integer to be identified later. Let $N>M K$ be an integer and suppose that $x, y, z$ are words such that $x y^{N} z \in L_{n}[\alpha]^{*}$. The result follows from the claim that, for sufficiently large $K$, depending only on $\alpha$ and $n, x y^{N+1} z$ belongs to $L_{n}[\alpha]^{*}$.

To prove the claim, we start with a factorization $x y^{N} z=w_{1} \ldots w_{m}$ where each $w_{j} \in L_{n}[\alpha]$. Consider each product of $M$ consecutive $y$ s within the factor $y^{N}$. If at least one of the factors appears completely within one of the $w_{j}$, then we have a factorization $w_{j}=x^{\prime} y^{M} z^{\prime}$ as indicated in Figure 3. In particular, the word $x^{\prime} y^{M} z^{\prime}$ belongs to the star-free language $L_{n}[\alpha]$. By the choice of $M$, we deduce that $w_{j}^{\prime}=x^{\prime} y^{M+1} z^{\prime} \in L_{n}[\alpha]$. Hence, for $p$ as in Figure 3,

$$
x y^{N+1} z=x y^{p} \cdot y^{M+1} \cdot y^{N-M-p} z=w_{1} \ldots w_{j-1} w_{j}^{\prime} w_{j+1} \ldots w_{m}
$$

is again a word from $L_{n}[\alpha]^{m}$, independently of the value of $K \geqslant 1$.

We may therefore assume that no factor $y^{M}$ appears completely within some factor $w_{j}$. Thus, each of the first $K<N / M$ consecutive factors $y^{M}$, which form a prefix of $y^{N}$, as well 


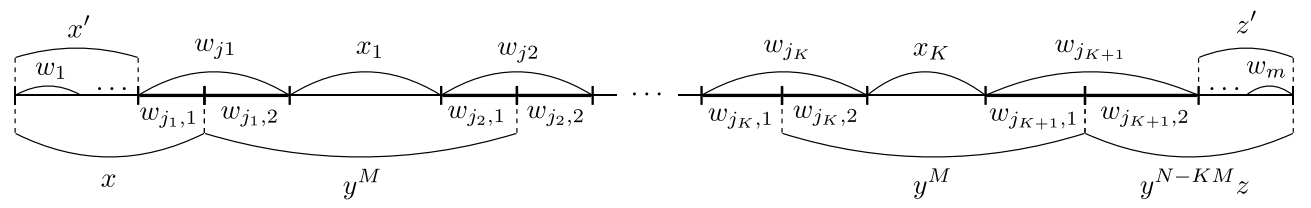

Figure 4 . The case where each $y^{M}$ overlaps several $w_{j}$.

as the product $y^{N-K M} z$, start in a different $w_{j}$, say in $w_{j_{1}}, \ldots, w_{j_{K+1}}$, with $j_{1}<\ldots<j_{K+1}$. This determines factorizations

$$
\begin{gathered}
w_{j_{s}}=w_{j_{s}, 1} w_{j_{s}, 2}, \\
y^{M}=w_{j_{s}, 2} x_{s} w_{j_{s+1}, 1} \quad(s=1, \ldots, K), \\
x=x^{\prime} w_{j_{1}, 1}, \\
y^{N-K M} z=w_{j_{K+1}, 2} z^{\prime},
\end{gathered}
$$

where each $x_{s}, x^{\prime}$, and $z^{\prime}$ is a word from $L_{n}[\alpha]^{*}$, as represented in Figure 4.

Consider a finite deterministic automaton recognizing the language $L_{n}[\alpha]$. Each pair of words $\left(w_{j_{s}, 1}, w_{j_{s}, 2}\right)$ determines two consecutive paths leading from the initial state to a final state. Thus, if $K$ is greater than the number of states, then there exist two indices $p, q$ such that $1 \leqslant p<q \leqslant K$ and the words $w_{j_{p}, 1}$ and $w_{j_{q}, 1}$ both lead from the initial state to the same state. It follows that $w_{j_{q}, 1} w_{j_{p}, 2}$ belongs to $L_{n}[\alpha]$. Hence the word

$$
\begin{aligned}
w_{j_{p+1}, 1} y^{M(q-p-2)} w_{j_{q-1}, 2} x_{q-1} w_{j_{q}, 1} \cdot w_{j_{p}, 2} x_{p} & =w_{j_{p+1}, 1} y^{M(q-p-1)} w_{j_{p}, 2} x_{p} \\
& =\left(w_{j_{p+1}, 1} w_{j_{p}, 2} x_{p}\right)^{q-p}
\end{aligned}
$$

belongs to $L_{n}[\alpha]^{*}$ where, for the second equality, we use the factorization (6.2) with $s=p$ for each $y^{M}$. Now $w_{j_{p+1}, 1} w_{j_{p}, 2} x_{p}$ is a conjugate of $y^{M}$ again by (6.2) and, therefore, it is of the form $t^{M}$, where $t$ is a conjugate of $y$. By Lemma $6.7, t$ belongs to $L_{n}[\alpha]^{*}$. On the other hand, note that

$$
\begin{aligned}
x y^{N} z & =x^{\prime} w_{j_{1}} x_{1} \ldots w_{j_{p-1}} x_{p-1} w_{j_{p}} x_{p} \cdot w_{j_{p+1}} x_{p+1} \ldots w_{j_{K}} x_{K} w_{j_{K+1}} z^{\prime}, \\
x y^{N+1} z & =x^{\prime} w_{j_{1}} x_{1} \ldots w_{j_{p-1}} x_{p-1} w_{j_{p}} x_{p} \cdot t \cdot w_{j_{p+1}} x_{p+1} \ldots w_{j_{K}} x_{K} w_{j_{K+1}} z^{\prime},
\end{aligned}
$$

where each of the factors separated by the dots belongs to $L_{n}[\alpha]^{*}$. Hence $x y^{N+1} z \in L_{n}[\alpha]^{*}$.

We are now in a position to complete the proof of our key result, namely that, for $\alpha$ in normal form and $n \geqslant \mu[\alpha]$, the languages $L_{n}[\alpha]$ are star-free.

Proof of Theorem 5.1. Let $i=\operatorname{rank}[\alpha]$. If $i=0$, then $L_{n}[\alpha]=\{\alpha\}$ is certainly a star-free language. We will therefore assume that $i \geqslant 1$. Let $\alpha=\gamma_{0}\left(\delta_{1}\right) \gamma_{1} \ldots\left(\delta_{r}\right) \gamma_{r}$ be the normal-form expression of $\alpha$.

We claim that each of the languages $L_{n}\left[\gamma_{0}\right], L_{n}\left[\delta_{j}\right]$ and $L_{n}\left[\delta_{j} \gamma_{j}\right](j=1, \ldots, r)$ is starfree. Since, by the definition of the normal form, each $\delta_{j}$ is primitive and in circular normal form, we deduce by Lemma 6.8 that $L_{n}\left[\delta_{j}\right]^{*}$ is star-free. In view of Lemma 3.1(c) and since the set of star-free languages is closed under concatenation, it follows that each language $L_{n}\left[\left(\delta_{j}\right) \gamma_{j}\right]=L_{n}\left[\delta_{j}\right]^{*} L_{n}\left[\delta_{j}\right]^{n-1} L_{n}\left[\delta_{j} \gamma_{j}\right]$ is also star-free. Taking Lemma 3.1(d) also into account, we conclude that the product

$$
L_{n}[\alpha]=L_{n}\left[\gamma_{0}\right] L_{n}\left[\left(\delta_{1}\right) \gamma_{1}\right] \ldots L_{n}\left[\left(\delta_{r}\right) \gamma_{r}\right]
$$

is star-free, as stated in the theorem. 
To prove the claim, we proceed by induction on $i \geqslant 1$. The case $i=1$ is immediate since then all the $\gamma_{j}$ and $\delta_{j}$ are words in $X^{*}$. Suppose that $i \geqslant 2$ and assume inductively that the claim holds for $\omega$-terms of rank less than $i$. Consider the $\omega$-term $\alpha^{\prime}=\gamma_{0} \delta_{1} \delta_{1} \gamma_{1} \ldots \delta_{r} \delta_{r} \gamma_{r}$. By condition (c) of the definition of an $\omega$-term in normal form, $\alpha^{\prime}$ is in normal form. By Lemma 3.3, since $\alpha^{\prime} \in E_{2}[\alpha]$, we have $\mu[\alpha] \geqslant \mu\left[\alpha^{\prime}\right]$. Hence $n \geqslant \mu\left[\alpha^{\prime}\right]$ and we may apply the induction hypothesis to the $\omega$-term $\alpha^{\prime}$ of rank $i-1 \geqslant 1$. Since $\alpha$ is in normal form and the $\omega$-terms $\delta_{j}$ are Lyndon words of positive rank, the first letter of each $\delta_{j}$ is the opening parenthesis of an $\omega$-subterm of highest (and positive) rank. Hence, if $\alpha^{\prime}=u_{0}\left(v_{1}\right) u_{1} \ldots\left(v_{s}\right) u_{s}$ is the normal-form expression of $\alpha^{\prime}$, then each factor $\gamma_{0}, \delta_{j}, \delta_{j} \gamma_{j}(j=1, \ldots, r)$ must be a product of some of the factors $u_{0},\left(v_{k}\right),\left(v_{k}\right) u_{k}(k=1, \ldots, s)$. By the induction hypothesis, each of the languages $L_{n}\left[u_{0}\right], L_{n}\left[v_{k}\right]$, and $L_{n}\left[v_{k} u_{k}\right](k=1, \ldots, s)$ is star-free. By the above argument, it follows that so are the languages $L_{n}\left[u_{0}\right], L_{n}\left[\left(v_{k}\right)\right]$, and $L_{n}\left[\left(v_{k}\right) u_{k}\right](k=1, \ldots, s)$. Finally, by Lemma 3.1(c), we deduce that each of the languages $L_{n}\left[\gamma_{0}\right], L_{n}\left[\delta_{j}\right], L_{n}\left[\delta_{j} \gamma_{j}\right](j=1, \ldots, r)$ is star-free, thus proving the induction step. This proves the claim and completes the proof of Theorem 5.1.

We do not know whether the bound $n \geqslant \mu[\alpha]$ is optimal but we do know that some bound is required, that is, that $L_{n}[\alpha]$ may not be star-free for $\alpha$ in normal form. An example is obtained by taking $\alpha=\left((a) a b(b) a^{2} b^{2}\right)$, where $a$ and $b$ are letters. Then $L_{1}[\alpha] \cap\left[a^{2} b^{2}\right]^{*}=\left[a^{2} b^{2} a^{2} b^{2}\right]^{+}$so that $L_{1}[\alpha]$ is not star-free since $\left[a^{2} b^{2}\right]^{*}$ is star-free and $\left[a^{2} b^{2} a^{2} b^{2}\right]^{+}$is not.

\section{Factors of $\omega$-words over A}

In this section we present further properties of the languages $L_{n}[\alpha]$ and derive some applications. The main result of this section is that every factor of an $\omega$-word over $A$ is also an $\omega$-word over A.

Recall that, given a pseudovariety $\mathrm{V}$, a finite semigroup $T \in \mathrm{V}$ satisfies the pseudoidentity $u=v$, with $u, v \in \bar{\Omega}_{X} \vee$, if, for every continuous homomorphism $\varphi: \bar{\Omega}_{X} \vee \rightarrow T$, we have $\varphi(u)=\varphi(v)$. For a finite semigroup $T$, let $\operatorname{ind}(T)$ be the smallest $\ell \geqslant 1$ such that for some $k \geqslant 1$ and every $s \in T$, we have $s^{\ell+k}=s^{\ell}$. Equivalently, $\operatorname{ind}(T)$ is the minimum positive integer $\ell$ such that $T$ satisfies the pseudoidentity $x^{\omega+\ell}=x^{\ell}$. Note that $\operatorname{ind}(T) \leqslant|T|$. We begin by proving that finite aperiodic semigroups do not separate an $\omega$-term from its expansions of sufficiently large exponent.

Lemma 7.1. Let $\alpha \in \mathcal{T}_{X}$ be an $\omega$-term and let $T \in \mathrm{A}$. If $n \geqslant \operatorname{ind}(T)$ and $w \in L_{n}[\alpha]$, then $T$ satisfies the pseudoidentity $\epsilon[\alpha]=w$.

Proof. Let $\varphi: \bar{\Omega}_{X} \mathrm{~A} \rightarrow T$ be a continuous homomorphism. Since $n \geqslant \operatorname{ind}(T)$, for every $m \geqslant n$, the semigroup $T$ satisfies the identity $x^{m}=x^{n}$. Hence, for every word $w \in L_{n}[\alpha]$, we have $\varphi(w)=\varphi(u)$, where $u$ is the word which is obtained from $\alpha$ by replacing all occurrences of the $\omega$ exponent by $n$.

Recall from $\S 2.2$ that the topological closures $\operatorname{cl}(L)$ and $\operatorname{cl}_{\mathrm{A}}(L)$ of a language $L$ in $\bar{\Omega}_{X} \mathrm{~S}$ and $\bar{\Omega}_{X} \mathrm{~A}$, respectively, are such that $p_{\mathrm{A}}(\operatorname{cl}(L))=\operatorname{cl}_{\mathrm{A}}(L)$. The following consequence of Lemma 7.1 will be useful.

Corollary 7.2. If $\alpha \in \mathcal{T}_{X}$ is an arbitrary $\omega$-term, then

$$
p_{\mathrm{A}}\left(\bigcap_{n} \operatorname{cl}\left(L_{n}[\alpha]\right)\right)=\{\epsilon[\alpha]\}=\bigcap_{n} p_{\mathrm{A}}\left(\operatorname{cl}\left(L_{n}[\alpha]\right)\right) .
$$

Proof. Denote by $\partial$ the unique homomorphism of unary semigroups $\mathcal{T}_{X} \rightarrow \bar{\Omega}_{X} \mathrm{~S}$ extending the identity mapping on $X$ so that $\epsilon=p_{\mathrm{A}} \circ \partial$. First note that, since $\partial[\alpha] \in \operatorname{cl}\left(L_{n}[\alpha]\right)$ for every 
$n$, certainly $\epsilon[\alpha] \in p_{\mathrm{A}}\left(\bigcap_{n} \operatorname{cl}\left(L_{n}[\alpha]\right)\right)$, so

$$
\{\epsilon[\alpha]\} \subseteq p_{\mathrm{A}}\left(\bigcap_{n} \operatorname{cl}\left(L_{n}[\alpha]\right)\right) \subseteq \bigcap_{n} p_{\mathrm{A}}\left(\operatorname{cl}\left(L_{n}[\alpha]\right)\right) .
$$

Let $v \in \bigcap_{n} p_{\mathrm{A}}\left(\operatorname{cl}\left(L_{n}[\alpha]\right)\right)$. For a continuous homomorphism $\psi: \bar{\Omega}_{X} \mathrm{~A} \rightarrow T$ onto a finite aperiodic semigroup $T$, let $\varphi=\psi \circ p_{\mathrm{A}}: \bar{\Omega}_{X} \mathrm{~S} \rightarrow T$ and choose any $n \geqslant \operatorname{ind}(T)$. Then

$$
\psi(v) \in \varphi\left(\operatorname{cl}\left(L_{n}[\alpha]\right)\right)=\varphi\left(L_{n}[\alpha]\right)=\{\varphi(\partial[\alpha])\}
$$

where the first equality follows from the continuity of $\varphi$ and the finiteness of $T$, and the second equality is a consequence of Lemma 7.1. Since $\bar{\Omega}_{X} A$ is residually in A, it follows that $v=\epsilon[\alpha]$.

We also have the following stronger result for $\omega$-terms in normal form.

Theorem 7.3. Let $w \in \Omega_{X}^{\omega} \mathrm{A}$ and let $\alpha$ be the normal-form representation of $w$. Then

$$
p_{\mathrm{A}}^{-1}(w)=\bigcap_{n} \operatorname{cl}\left(L_{n}[\alpha]\right)
$$

Proof. The inclusion $\bigcap_{n} \operatorname{cl}\left(L_{n}[\alpha]\right) \subseteq p_{\mathrm{A}}^{-1}(w)$ follows from Corollary 7.2. For the reverse inclusion, assuming that $v \in \bar{\Omega}_{X} \mathrm{~S}$ is such that $p_{\mathrm{A}}(v)=w$, we have $p_{\mathrm{A}}(v) \in p_{\mathrm{A}}\left(\operatorname{cl}\left(L_{n}[\alpha]\right)\right)$ for all $n$. Let $\left(v_{n}\right)_{n}$ be a sequence of words converging to $v$ in $\bar{\Omega}_{X} \mathrm{~S}$. Then $\lim v_{n}=w$ in $\bar{\Omega}_{X} \mathrm{~A}$ and so, since by Theorem 5.1 the set $p_{\mathrm{A}}\left(\operatorname{cl}\left(L_{n}[\alpha]\right)\right)$ is open and contains $w$, by taking a suitable subsequence we may assume that $v_{n} \in p_{\mathrm{A}}\left(\operatorname{cl}\left(L_{n}[\alpha]\right)\right) \cap X^{+}=L_{n}[\alpha]$. Since $\left(L_{n}[\alpha]\right)_{n}$ is a decreasing sequence of languages, it follows that $v \in \operatorname{cl}\left(L_{n}[\alpha]\right)$ for all $n$.

We now prove the main result of this section which does not apparently follow easily from McCammond's results.

Theorem 7.4. If $v \in \Omega_{X}^{\omega} \mathrm{A}$ and $u \in \bar{\Omega}_{X} \mathrm{~A}$ is a factor of $v$, then $u \in \Omega_{X}^{\omega} \mathrm{A}$.

Proof. By symmetry, it suffices to prove the result when $u$ is a prefix of $v$, that is, when there exists $w \in \bar{\Omega}_{X} \mathrm{~A}$ such that $u w=v$. Let $\alpha$ be the normal-form representation of $v$. We proceed by induction on $\operatorname{rank}[\alpha]$. We assume inductively that the result holds for all elements of $\Omega_{X}^{\omega} \mathrm{A}$ with rank strictly smaller than $\operatorname{rank}[\alpha]$.

Since $L_{n}[\alpha]$ is star-free for $n \geqslant \mu[\alpha]$ by Theorem 5.1 , its closure $\operatorname{cl}_{\mathrm{A}}\left(L_{n}[\alpha]\right)$ is an open subset of $\bar{\Omega}_{X} \mathrm{~A}$. Hence, there exist sequences $\left(u_{m}\right)_{m}$ and $\left(w_{m}\right)_{m}$ converging to $u$ and $w$ respectively such that $u_{n} w_{n} \in L_{n}[\alpha]$ for all $n \geqslant \mu[\alpha]$.

As an $\omega$-term, $\alpha$ admits a unique factorization in the semigroup $\mathcal{T}_{X}$ of the form $\alpha=$ $x_{0} x_{1} x_{2} \ldots x_{2 p-1} x_{2 p}$, where each $x_{2 i}$ is a finite word and each $x_{2 i-1}$ is an $\omega$-term of the form $x_{2 i-1}=\left(y_{2 i-1}\right)$. Note that we include here the case where $\alpha$ is a word, for which $p=0$. Since $\alpha$ is in normal form, each $y_{2 i-1}$ is an $\omega$-term of rank less than rank $[\alpha]$ (although not necessarily of $\operatorname{rank}[\alpha]-1)$. In view of Lemma 3.1 and each relation $u_{n} w_{n} \in L_{n}[\alpha]$, there is a 'cutting' index $c_{n} \in\{0, \ldots, 2 p\}$ and there are factorizations $u_{n}=u_{n}^{\prime} u_{n}^{\prime \prime}$ and $w_{n}=w_{n}^{\prime} w_{n}^{\prime \prime}$ such that

$$
u_{n}^{\prime} \in L_{n}\left[x_{0} \ldots x_{c_{n}-1}\right], \quad u_{n}^{\prime \prime} w_{n}^{\prime} \in L_{n}\left[x_{c_{n}}\right], \quad w_{n}^{\prime \prime} \in L_{n}\left[x_{c_{n}+1} \ldots x_{2 p}\right] .
$$

Since the number of possible cutting indices depends only on $\alpha$ and not on $n$, there is a strictly increasing sequence of indices $\left(n_{k}\right)_{k}$ whose corresponding cutting indices are all equal to a certain fixed $c$. By compactness of $\bar{\Omega}_{X} \mathrm{~A}$, one may further assume that the sequences $\left(u_{n_{k}}^{\prime}\right)_{k},\left(u_{n_{k}}^{\prime \prime}\right)_{k},\left(w_{n_{k}}^{\prime}\right)_{k}$, and $\left(w_{n_{k}}^{\prime \prime}\right)_{k}$ converge to, say, $u^{\prime}, u^{\prime \prime}, w^{\prime}, w^{\prime \prime}$ respectively. By continuity 
of multiplication, and since $\left(L_{n}[\beta]\right)_{n}$ is a decreasing sequence of languages for every $\omega$-term $\beta$, it follows that

$$
\begin{gathered}
u^{\prime} \in \bigcap_{n} \operatorname{cl}_{\mathrm{A}}\left(L_{n}\left[x_{0} \ldots x_{c-1}\right]\right), \\
u^{\prime \prime} w^{\prime} \in \bigcap_{n} \operatorname{cl}_{\mathrm{A}}\left(L_{n}\left[x_{c}\right]\right), \\
w^{\prime \prime} \in \bigcap_{n} \operatorname{cl}_{\mathrm{A}}\left(L_{n}\left[x_{c+1} \ldots x_{2 p}\right]\right) .
\end{gathered}
$$

By Corollary 7.2, the preceding intersections are reduced to the $\omega$-words $\epsilon\left[x_{0} \ldots x_{c-1}\right], \epsilon\left[x_{c}\right]$, and $\epsilon\left[x_{c+1} \ldots x_{2 p}\right]$, respectively. Hence $u^{\prime}, w^{\prime \prime} \in \Omega_{X}^{\omega} \mathrm{A}$ and $u^{\prime \prime} w^{\prime}=\epsilon\left[x_{c}\right]$. If $c$ is even, then $u^{\prime \prime}$ is a prefix of the word $x_{c}$ and hence $u=u^{\prime} u^{\prime \prime} \in \Omega_{X}^{\omega} \mathrm{A}$, as required. Hence we may as well assume that $\alpha$ is of the form $\alpha=(y)$.

By Lemma 3.1(e), we have $L_{n}[\alpha]=L_{n}\left[y^{n}\right] L_{n}[y]^{*}$. Thus, in view of the relation $u_{n} w_{n} \in L_{n}[\alpha]$, there exist factorizations $u_{n}=u_{n}^{\prime} u_{n}^{\prime \prime}$ and $w_{n}=w_{n}^{\prime} w_{n}^{\prime \prime}$ such that $u_{n}^{\prime} \in L_{n}\left[y^{r_{n}}\right], u_{n}^{\prime \prime} w_{n}^{\prime} \in L_{n}[y]$, and $w_{n}^{\prime \prime} \in L_{n}\left[y^{s_{n}}\right]$, with $r_{n}+s_{n}+1 \geqslant n$. Suppose that there is a strictly increasing sequence of indices $\left(n_{k}\right)_{k}$ such that $r_{n_{k}}=r$ is constant. We may assume that the sequences $\left(u_{n_{k}}^{\prime}\right)_{k}$, $\left(u_{n_{k}}^{\prime \prime}\right)_{k},\left(w_{n_{k}}^{\prime}\right)_{k}$, and $\left(w_{n_{k}}^{\prime \prime}\right)_{k}$ converge to, say, $u^{\prime}, u^{\prime \prime}, w^{\prime}, w^{\prime \prime}$ respectively. As above, it follows that $u^{\prime}, w^{\prime \prime} \in \Omega_{X}^{\omega} \mathrm{A}$ and $u^{\prime \prime} w^{\prime}=\epsilon[y]$. Since $\operatorname{rank}[y]<\operatorname{rank}[\alpha]$, the induction hypothesis then implies that $u^{\prime \prime}$ is an $\omega$-term and, therefore so is $u=u^{\prime} u^{\prime \prime}$.

Hence we may assume that $r_{n} \rightarrow \infty$ as $n \rightarrow \infty$. This implies that $y^{r_{n}} \rightarrow(y)$ in $\Omega_{X}^{\omega} \mathrm{A}$. Assuming again that $\left(u_{n_{k}}^{\prime}\right)_{k},\left(u_{n_{k}}^{\prime \prime}\right)_{k},\left(w_{n_{k}}^{\prime}\right)_{k}$, and $\left(w_{n_{k}}^{\prime \prime}\right)_{k}$ converge to $u^{\prime}, u^{\prime \prime}, w^{\prime}, w^{\prime \prime}$ respectively, we conclude that $u^{\prime}=\epsilon[(y)] \in \Omega_{X}^{\omega} \mathrm{A}$ and $u^{\prime \prime} w^{\prime}=\epsilon[y]$. Invoking once more the induction hypothesis as above, the induction step is finally achieved, which proves the theorem.

Note that Theorem 5.1 only intervenes in the above proof to show that $\operatorname{cl}_{A}\left(L_{n}[\alpha]\right)$ is an open subset of $\bar{\Omega}_{X} \mathrm{~A}$, a property which in fact is equivalent to $L_{n}[\alpha]$ being star-free.

Some applications of Theorem 7.4 can be found in [9]. It plays, in particular, an important role in establishing the main result of that paper, namely a characterization of pseudowords over A which are given by $\omega$-terms. Other applications of Theorem 7.4 and of properties of the languages $L_{n}[\alpha]$, such as an algorithm to compute the closure $\mathrm{cl}_{\mathrm{A}}(L)$ of a regular language $L$, have been published in [8].

\section{References}

1. J. Almeida, 'Implicit operations on finite $\mathcal{J}$-trivial semigroups and a conjecture of I. Simon', J. Pure Appl. Algebra 69 (1990) 205-218.

2. J. Almeida, Finite semigroups and universal algebra (World Scientific, Singapore, 1995).

3. J. Almeida, 'Hyperdecidable pseudovarieties and the calculation of semidirect products', Int. J. Algebra Comput. 9 (1999) 241-261.

4. J. Almeida, 'Some algorithmic problems for pseudovarieties', Publ. Math. Debrecen 54(suppl.) (1999) 531-552; Automata and formal languages, VIII (Salgótarján, 1996).

5. J. Almeida, 'Profinite semigroups and applications', Structural theory of automata, semigroups, and universal algebra: Proceedings of the NATO Advanced Study Institute on Structural Theory of Automata, Semigroups and Universal Algebra, Montréal, Québec, Canada, 7-18 July 2003, NATO Science Series II: Mathematics, Physics and Chemistry 207 (eds V. B. Kudryavtsev and I. G. Rosenberg; Springer, New York, 2005).

6. J. Almeida, J. C. Costa and M. Zeitoun, 'Tameness of pseudovariety joins involving R', Monatsh. Math. 146 (2005) no. 2, 89-111.

7. J. Almeida, J. C. Costa and M. Zeitoun, 'Complete reducibility of systems of equations with respect to R', Port. Math. 64 (2007) 445-508.

8. J. Almeida, J. C. Costa and M. Zeitoun, 'Closures of regular languages for profinite topologies', Semigroup Forum 89 (2014) 20-40.

9. J. Almeida, J. C. Costa and M. Zeitoun, 'Iterated periodicity over finite aperiodic semigroups', Eur. J. Combin. 37 (2014) 115-149. 
10. J. Almeida and B. Steinberg, 'On the decidability of iterated semidirect products and applications to complexity', Proc. London Math. Soc. (3) 80 (2000) 50-74.

11. J. Almeida and B. Steinberg, 'Syntactic and global semigroup theory, a synthesis approach', Algorithmic problems in groups and semigroups (eds J. C. Birget, S. W. Margolis, J. Meakin and M. V. Sapir; Birkhäuser, Boston, 2000) 1-23.

12. J. Almeida and M. Zeitoun, 'An automata-theoretic approach to the word problem for $\omega$-terms over R', Theoret. Comput. Sci. 370 (2007) 131-169.

13. J. C. Costa, 'Free profinite locally idempotent and locally commutative semigroups', J. Pure Appl. Algebra $163(2001)$ 19-47.

14. J. C. Costa, 'Canonical forms for free $\kappa$-semigroups', Discrete Math. Theor. Comput. Sci. 16 (2014) no. 1 , $159-178$.

15. J. C. Costa and C. Nogueira, 'Complete reducibility of the pseudovariety LSI', Int. J. Algebra Comput. 19 (2009) 1-36.

16. J. C. Costa and M. L. Teixeira, 'Tameness of the pseudovariety LSI', Int. J. Algebra Comput. 14 (2004) $627-654$.

17. K. Henckell, 'Pointlike sets: the finest aperiodic cover of a finite semigroup', J. Pure Appl. Algebra 55 (1988) no. 1-2, 85-126.

18. M. Huschenbett and M. Kufleitner, 'Ehrenfeucht-Fraïssé games on omega-terms', 31st International Symposium on Theoretical Aspects of Computer Science (STACS 2014), Leibniz International Proceedings in Informatics (LIPIcs) 25 (eds E. W. Mayr and N. Portier; Schloss Dagstuhl-Leibniz-Zentrum für Informatik, Dagstuhl, 2014) 374-385.

19. M. Lothaire, Combinatorics on words (Addison-Wesley, Reading, MA, 1983).

20. M. Lothaire, Algebraic combinatorics on words (Cambridge University Press, Cambridge, 2002).

21. J. MCCAMmond, 'The solution to the word problem for the relatively free semigroups satisfying $t^{a}=t^{a+b}$ with $a \geqslant 6$ ', Int. J. Algebra Comput. 1 (1991) 1-32.

22. J. McCammond, 'Normal forms for free aperiodic semigroups', Int. J. Algebra Comput. 11 (2001) 581-625.

23. R. MCNaughton and S. PApert, Counter-free automata (MIT Press, Cambridge, MA, 1971).

24. A. MourA, 'The word problem for $\omega$-terms over DA', Theoret. Comput. Sci. 412 (2011) no. 46, 6556-6569.

25. T. Place and M. Zeitoun, 'Separating regular languages with first-order logic', CSL-LICS '14: Proceedings of the Joint Meeting of the Twenty-Third EACSL Annual Conference on Computer Science Logic (CSL) and the Twenty-Ninth Annual ACM/IEEE Symposium on Logic in Computer Science (LICS) (ACM Press, New York, 2014).

26. J. Rhodes and B. Steinberg, The q-theory of finite semigroups, Monographs in Mathematics (Springer, New York, 2009).

27. M. P. Schützenberger, 'On finite monoids having only trivial subgroups', Inform. and Control 8 (1965) 190-194.

\section{J. Almeida CMUP}

Departamento de Matemática

Faculdade de Ciências

Universidade do Porto

Rua do Campo Alegre 687

4169-007 Porto

Portugal

jalmeida@fc.up.pt

\section{Zeitoun}

LaBRI

Université Bordeaux \& CNRS UMR 5800

351 cours de la Libération

33405 Talence Cedex

France

mz@labri.fr

\author{
J. C. Costa \\ CMAT \\ Departamento de Matemática e \\ Aplicações \\ Universidade do Minho \\ Campus de Gualtar \\ 4700-320 Braga \\ Portugal
}

jcosta@math.uminho.pt 